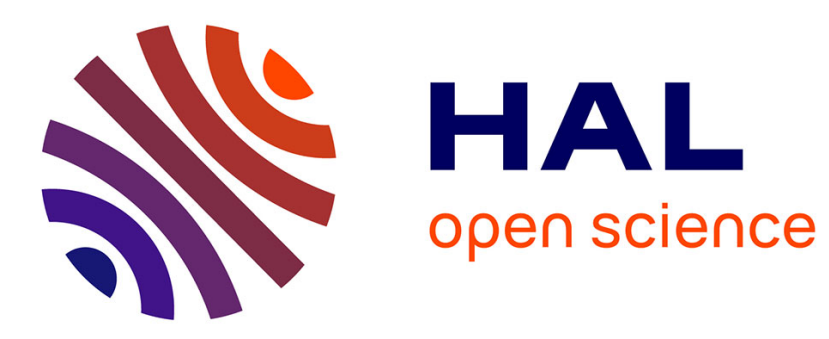

\title{
Méthodes numériques avancées en vibroacoustique basses et moyennes fréquences
}

Roger Ohayon, Christian Soize

\section{To cite this version:}

Roger Ohayon, Christian Soize. Méthodes numériques avancées en vibroacoustique basses et moyennes fréquences. Revue Européenne des Éléments Finis, 1999, 8 (5-6), pp.607-637. 10.1080/12506559.1999.10511399 . hal-00765801

\section{HAL Id: hal-00765801 https://hal.science/hal-00765801}

Submitted on 16 Dec 2012

HAL is a multi-disciplinary open access archive for the deposit and dissemination of scientific research documents, whether they are published or not. The documents may come from teaching and research institutions in France or abroad, or from public or private research centers.
L'archive ouverte pluridisciplinaire HAL, est destinée au dépôt et à la diffusion de documents scientifiques de niveau recherche, publiés ou non, émanant des établissements d'enseignement et de recherche français ou étrangers, des laboratoires publics ou privés. 


\title{
Méthodes numériques avancées en vibroacoustique basses et moyennes fréquences
}

\author{
Roger Ohayon* - Christian Soize ${ }^{* *}$ \\ * CNAM/LMSS, Laboratoire de Mécanique des Structures et des \\ Systèmes Couplés, 2 rue Conté, F-75003 Paris \\ ohayon@cnam.fr \\ ** ONERA/DDSS, Département Dynamique des Structures et des \\ Systèmes Couplés, BP 72, F-92322 Châtillon Cedex \\ soize@onera.fr
}

\begin{abstract}
RÉSUMÉ. Ce papier présente une synthèse des modélisations et des méthodes numériques associées pour la prévision des vibrations linéaires des systèmes vibroacoustiques tridimensionnels quelconques constitués d'une structure contenant un fuide acoustique interne dissipatif et plongée dans un fuide acoustique externe parfait non borné. On tient compte d'impédances acoustiques de parois dans la modélisation. Concernant la vibroacoustique BF, on présente les modèles matriciels réduits basés sur la projection de Ritz-Galerkin du modèle éléments fnis en utilisant des bases modales adaptées. Enf $n$ en vibroacoustique $M F$, on présente la modélisation éléments fnis et les méthodes de résolution, c'est-à-dire, une méthode à deux échelles de temps et une méthode de construction de modèles matriciels réduits adaptés.

ABSTRACT. This paper presents a synthesis of modeling and associated numerical methods for the prediction of linear vibrations of arbitrary structural-acoustic tridimensional systems constituted of a structure containing an internal dissipative acoustic fuid and surrounded by an external inviscid acoustic fuid occupying an unbounded domain. The model includes wall acoustic impedances. For low-frequency vibroacoustic problems, we present reduced matrix models based on RitzGalerkin projection of the fnite element model using appropriate modal basis. Finally, for medium-frequency vibroacoustic problems, we present the fnite element model and solving methods, i.e. a two time scales procedure and a method of construction of appropriate reduced matrix models.
\end{abstract}

MOTS-CLÉS : Vibroacoustique, élastoacoustique, vibrations basses fréquences, vibrations moyennes fréquences, méthodes numériques, méthodes des éléments fnis, méthodes d'élements de frontière, modèles matriciels réduits.

KEYWORDS: Structural acoustics, vibroacoustics, low-frequency vibrations, medium-frequency vibrations, numerical methods, fnite element method, boundary element method, reduced matrix models. 


\section{Introduction}

Nous présentons une synthèse concernant les modélisations et les méthodes numériques associées pour la prévision des vibrations linéaires des systèmes vibroacoustiques tridimensionnels quelconques. Un système vibroacoustique est constitué d'une structure tridimensionnelle couplée à un f uide acoustique interne dissipatif (gaz ou liquide) occupant un domaine borné de l'espace (cavité acoustique) et couplée à un f uide acoustique externe parfait (gaz ou liquide) occupant un domaine non borné de l'espace. Sur une partie de l'interface entre la structure et la cavité acoustique interne, on considère un milieu ayant des propriétés acoustiques, modélisé par une impédance de paroi. Nous ne considérons ici que des structures sans complexité structurale (la complexité structurale étant due à la présence de nombreuses sous-structures secondaires : équipements, etc.) et nous renvoyons le lecteur à [OHA 98] pour le cas général d'une structure principale accessible à une modélisation déterministe classique couplée à une complexité structurale relevant d'une modélisation probabiliste.

Dans le paragraphe 2, nous rappelons les problématiques basses et moyennes fréquences pour les systèmes vibroacoustiques et nous introduisons la stratégie numérique recommandée pour chacune des deux bandes. Il est à noter que la bande des hautes fréquences ne relève pas des méthodes numériques (voir paragraphe 2).

Puis dans le paragraphe 3 , nous présentons le problème à résoudre en basses et moyennes fréquences. Le système vibroacoustique est soumis à des excitations extérieures constituées d'une densité de source acoustique interne, d'une densité de source acoustique externe, d'une onde plane incidente dans le milieu extérieur et enf n de densités de force mécanique surfacique et volumique appliquées à la structure.

Le paragraphe 4 est consacré au problème acoustique externe, c'est-à-dire à la construction de l'opérateur de frontière d'impédance acoustique par une méthode d'équations intégrales de frontière sans fréquences irrégulières et de l'opérateur de rayonnement acoustique. Le premier est utilisé pour écrire le couplage de la structure avec le fuide acoustique externe aussi bien pour le domaine BF que pour le domaine MF. Le second permet de calculer le rayonnement acoustique dans le f uide extérieur en BF et MF dès que le problème couplé est résolu. La discrétisation par la méthode des éléments $\mathrm{f}$ nis de ces opérateurs intégraux (méthode des éléments $\mathrm{f}$ nis de frontière) conduit à une matrice symétrique complexe sans problèmes induits par les fréquences irrégulières.

Dans le paragraphe 5, on introduit la modélisation du système vibroacoustique adaptée au domaine BF et nous présentons d'une part, la discrétisation par éléments $\mathrm{f}$ nis et, d'autre part, le modèle matriciel réduit basé sur la projection de Ritz-Galerkin utilisant des bases modales adaptées.

Enf n, le dernier paragraphe est consacré à la modélisation du système vibroacoustique adaptée au domaine MF et nous présentons la discrétisation par éléments f nis et nous donnons des indications sur l'obtention de modèles matriciels réduits basés sur la projection de Ritz-Galerkin utilisant des bases adaptées au domaine MF (bases différentes de bases modales).

\section{Problématique des basses et des moyennes fréquences pour les systèmes vibroacoustiques complexes et stratégies numériques}

Les différents types de comportement vibratoire de systèmes vibroacoustiques conduisent à introduire trois domaines de fréquence (voir Figure 1) : domaines des Basses Fréquences (BF), des Moyennes Fréquences (MF) et des Hautes Fréquences (HF).

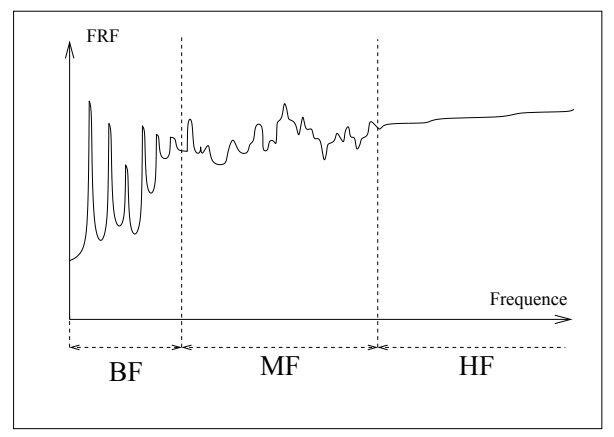


Figure 1. Fonction de Réponse en Fréquence (FRF) d'une composante de l'accélération en un point de la structure du système vibroacoustique : déf nition des trois domaines de fréquences

Le domaine BF (encore appelé le domaine modal) est déf ni classiquement comme le domaine pour lequel la structure couplée avec la cavité acoustique interne présente des résonances isolées (faible densité modale). Le domaine MF est déf ni comme le domaine intermédiaire entre les domaines BF et HF, pour lequel la structure couplée avec la cavité acoustique interne ne présente plus de résonances isolées mais présente encore de fortes irrégularités correspondant à de grandes variations de la densité modale (localement grande et localement faible dans la bande MF), les modes intervenant étant de rang élevé. Il est à noter que pour le domaine $\mathrm{MF}$, la géométrie et les conditions aux limites jouent, comme pour le domaine BF, un rôle fondamental. De plus, dans le domaine MF, une bonne modélisation de la dissipation au travers des lois de comportement est absolument nécessaire (il est à noter que s'il y avait une complexité structurale, elle induirait en plus une dissipation apparente dans la structure principale par transfert d'énergie mécanique vers les sous-structures de la complexité structurale, dissipation qui doit être modélisée). Il est important de signaler que le domaine MF n'existe pas pour des structures de forme géométrique simple telles qu'une poutre droite encastrée, une plaque rectangulaire simplement appuyée, une coque mince cylindrique, etc.

Concernant la structure couplée avec le f uide acoustique interne les formulations pour les domaines BF et MF ne sont pas les mêmes. Par contre, pour le fuide externe, on utilise la même formulation pour les deux domaines. En BF, la stratégie numérique consiste à ne pas résoudre fréquence par fréquence le système matriciel provenant de la discrétisation par éléments f nis mais à construire un modèle matriciel symétrique réduit en utilisant les modes de la structure in vacuo et les modes acoustiques de la cavité acoustique interne avec parois fxes. L'équation matricielle réduite est résolue fréquence par fréquence dans la bande BF. En MF, le système matriciel provenant de la discrétisation par éléments $\mathrm{f}$ nis étant de très grande taille, il ne peut pas être résolu fréquence par fréquence dans la bande MF. On recommande alors une méthode numérique à deux échelles de temps adaptée au domaine MF. En ce qui concerne la recherche de modèle réduit pour le domaine MF, l'approche BF n'étant plus pratiquable, une base de projection de Ritz-Galerkin appropriée doit être utilisée [OHA 98] et fait l'objet de recherches en cours [SOI 98].

\section{Pose du problème en basses et moyennes fréquences}

On considère les vibrations linéaires autour d'un état d'équilibre statique, supposé non précontraint pour simplif er, pris comme état de référence, d'un système couplé tridimensionnel constitué d'une structure élastique dissipative occupant un domaine borné $\Omega_{S}$ et qui contient un f uide acoustique interne dissipatif (gaz ou liquide, et dans le cas d'un liquide avec surface libre, les effets de gravité ne sont pas pris en compte) occupant un domaine borné $\Omega$. La formulation est écrite dans le domaine fréquentiel. Ce système est plongé dans un fuide acoustique externe parfait (gaz ou liquide) occupant le domaine non borné $\Omega_{E}$ (voir Figure 2).

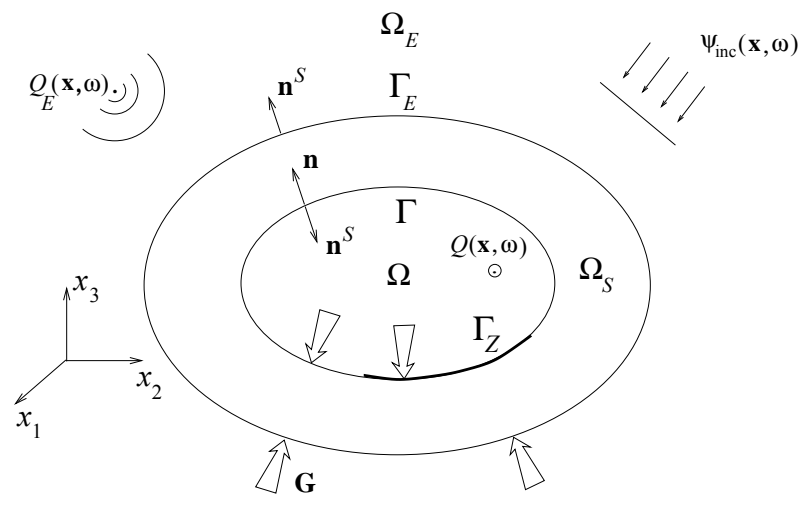

Figure 2. Conf guration du système vibroacoustique

La partie $\Gamma_{Z}$ du bord $\partial \Omega=\Gamma \cup \Gamma_{Z}$ de $\Omega$, a des propriétés acoustiques modélisées par une impédance de 
bord $Z(\mathbf{x}, \omega)$ à valeurs complexes et déf nie en tout point $\mathbf{x}$ de $\Gamma_{Z}$ et pour chaque pulsation $\omega$. Les excitations du système vibroacoustique sont respectivement, une densité de source acoustique interne $Q(\mathbf{x}, \omega)$ pour $\mathbf{x}$ dans $\Omega$, une densité de source acoustique externe $Q_{E}(\mathbf{x}, \omega)$ pour $\mathbf{x}$ dans $\Omega_{E}$, une onde plane incidente dans $\Omega_{E}$ déf nie par un potentiel de vitesse $\psi_{\text {inc }}(\mathbf{x}, \omega)=\psi_{0}(\omega) \exp \{-i \mathbf{k} \cdot \mathbf{x}\}$, une densité de forces surfaciques $\mathbf{G}(\mathbf{x}, \omega)$ pour $\mathbf{x}$ sur le bord $\partial \Omega_{S}=\Gamma \cup \Gamma_{Z} \cup \Gamma_{E}$ de $\Omega_{S}$ et enf $\mathbf{n}$ une densité de forces volumiques $\mathbf{g}(\mathbf{x}, \omega)$ pour $\mathbf{x}$ dans $\Omega_{S}$. Les inconnues sont le champ de déplacement $\mathbf{u}(\mathbf{x}, \omega)=\left(u_{1}(\mathbf{x}, \omega), u_{2}(\mathbf{x}, \omega), u_{3}(\mathbf{x}, \omega)\right)$ dans la structure $\Omega_{S}$, le champ de pression $p(\mathbf{x}, \omega)$ dans le f uide interne $\Omega$ et le champ de pression $p_{E}(\mathbf{x}, \omega)$ dans le f uide externe $\Omega_{E}$.

\section{Problème d'acoustique externe}

\subsection{Le problème de Neumann extérieur relatif à l'équation d'Helmholtz}

La géométrie est déf nie par la Figure 3.

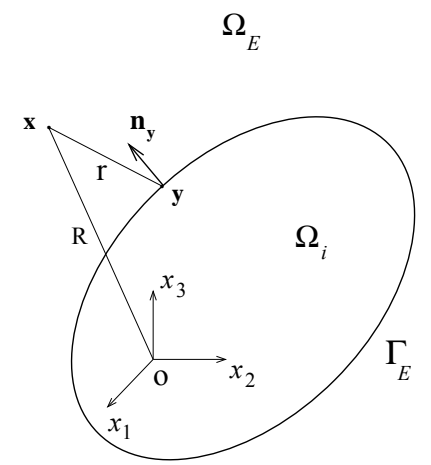

Figure 3. Géométrie du domaine fuide acoustique externe

Le f uide est parfait et est décrit par un champ inconnu scalaire ; on choisit pour ce problème extérieur le potentiel des vitesses $\psi(\mathbf{x}, \omega)$ en un point $\mathbf{x}$ de $\Omega_{E}$ et à la fréquence angulaire $\omega$, relié à la f uctuation de pression $p_{E}(\mathbf{x}, \omega)$ par l'équation

$$
p_{E}(\mathbf{x}, \omega)=-i \omega \rho_{E} \psi(\mathbf{x}, \omega) \quad \text { dans } \quad \Omega_{E},
$$

où $\rho_{E}$ est la masse volumique constante du f uide extérieur à l'équilibre. En désignant par $c_{E}$ la célérité constante du son dans le f uide à l'équilibre et en posant $k=\omega / c_{E}$, le problème de Neumann extérieur s'écrit :

$$
\begin{gathered}
\nabla^{2} \psi(\mathbf{x}, \omega)+k^{2} \psi(\mathbf{x}, \omega)=0 \quad \text { dans } \quad \Omega_{E}, \\
\frac{\partial \psi(\mathbf{y}, \omega)}{\partial \mathbf{n}_{\mathbf{y}}}=v(\mathbf{y}) \quad \text { sur } \quad \Gamma_{E}, \\
|\psi|=O\left(\frac{1}{R}\right), \quad\left|\frac{\partial \psi}{\partial R}+i k \psi\right|=O\left(\frac{1}{R^{2}}\right),
\end{gathered}
$$

avec $R=\|\mathbf{x}\| \rightarrow+\infty$, où $\partial / \partial R$ est la dérivée dans la direction radiale et où $v(\mathbf{y})$ est un champ de vitesse normale sur $\Gamma_{E}$ qui est donné. L'Eq. [4] correspond à la condition sortante de rayonnement à l'inf ni de Sommerfeld.

\subsection{Expression du champ de pression dans le fluide acoustique externe}

Pour tout $\omega \neq 0$ réel, le problème aux limites déf ni par les Eqs. [2] à [4] admet une unique solution. Le champ de pression $\left.p_{E}\right|_{\Gamma_{E}}(\omega)$ sur la paroi $\Gamma_{E}$ s'écrit alors en utilisant l'Eq. [1],

$$
\left.p_{E}\right|_{\Gamma_{E}}(\omega)=\mathbf{Z}_{\Gamma_{E}}(\omega) v,
$$


où $\mathbf{Z}_{\Gamma_{E}}(\omega)$ est l'opérateur de frontière d'impédance acoustique. Le champ de pression $p_{E}(\mathbf{x}, \omega)$ dans le f uide extérieur $\Omega_{E}$ s'écrit

$$
p_{E}(\mathbf{x}, \omega)=\mathbf{Z}_{\mathrm{rad}}(\mathbf{x}, \omega) v,
$$

où $\mathbf{Z}_{\mathrm{rad}}(\mathbf{x}, \omega)$ est l'opérateur d'impédance de rayonnement acoustique dans le f uide extérieur. On introduit

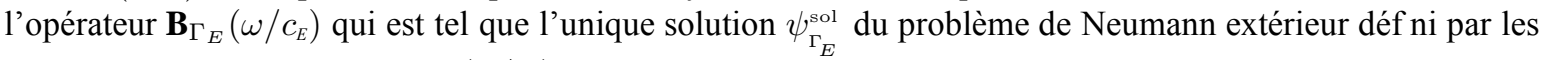
Eqs. [2] à [4] s'écrit $\psi_{\Gamma_{E}}^{\text {sol }}=\mathbf{B}_{\Gamma_{E}}\left(\omega / c_{E}\right) v$, ce qui montre en utilisant l'Eq. [1], que l'on a

$$
\mathbf{Z}_{\Gamma_{E}}(\omega)=-i \omega \rho_{E} \mathbf{B}_{\Gamma_{E}}\left(\omega / c_{E}\right)
$$

\subsection{Construction de l'opérateur de frontière d'impédance acoustique : Méthode d'équations intégrales de frontière symétrique sans fréquences irrégulières}

La formulation variationnelle qui permet de construire l'opérateur $\mathbf{B}_{\Gamma_{E}}\left(\omega / c_{E}\right)$ déf ni par l'Eq. [7] s'écrit (voir [OHA 98]) :

$$
\left[\begin{array}{c}
0 \\
\psi_{\Gamma_{E}}^{\text {sol }}
\end{array}\right]=\left[\begin{array}{cc}
-\mathbf{S}_{\mathrm{T}}\left(\omega / c_{E}\right) & \frac{1}{2}{ }^{t} \mathbf{I}-{ }^{t} \mathbf{S}_{\mathrm{D}}\left(\omega / c_{E}\right) \\
\frac{1}{2} \mathbf{I}-\mathbf{S}_{\mathrm{D}}\left(\omega / c_{E}\right) & \mathbf{S}_{\mathrm{S}}\left(\omega / c_{E}\right)
\end{array}\right]\left[\begin{array}{c}
\psi_{\Gamma_{E}} \\
v
\end{array}\right] .
$$

Les opérateurs intégraux $\mathbf{S}_{\mathrm{S}}\left(\omega / c_{E}\right), \mathbf{S}_{\mathrm{D}}\left(\omega / c_{E}\right)$ et $\mathbf{S}_{\mathrm{T}}\left(\omega / c_{E}\right)$ sont déf nis par les formes bilinéaires complexes

$$
\begin{gathered}
<\mathbf{S}_{\mathrm{S}}\left(\omega / c_{E}\right) v, \delta v>=\int_{\Gamma_{E}} \int_{\Gamma_{E}} G(\mathbf{x}-\mathbf{y}) v(\mathbf{y}) \delta v(\mathbf{x}) d s_{\mathbf{y}} d s_{\mathbf{x}} . \\
<\mathbf{S}_{\mathrm{D}}\left(\omega / c_{E}\right) \psi_{\Gamma_{E}}, \delta v>=\int_{\Gamma_{E}} \int_{\Gamma_{E}} \frac{\partial G(\mathbf{x}-\mathbf{y})}{\partial \mathbf{n}_{\mathbf{y}}} \psi_{\Gamma_{E}}(\mathbf{y}) \delta v(\mathbf{x}) d s_{\mathbf{y}} d s_{\mathbf{x}} . \\
<\mathbf{S}_{\mathrm{T}}\left(\omega / c_{E}\right) \psi_{\Gamma_{E}}, \delta \psi_{\Gamma_{E}}>=-k^{2} \int_{\Gamma_{E}} \int_{\Gamma_{E}} G(\mathbf{x}-\mathbf{y}) \mathbf{n}_{\mathbf{x}} \cdot \mathbf{n}_{\mathbf{y}} \psi_{\Gamma_{E}}(\mathbf{y}) \delta \psi_{\Gamma_{E}}(\mathbf{x}) d s_{\mathbf{y}} d s_{\mathbf{x}} \\
+\int_{\Gamma_{E}} \int_{\Gamma_{E}} G(\mathbf{x}-\mathbf{y})\left\{\mathbf{n}_{\mathbf{y}} \times \nabla_{\mathbf{y}} \psi_{\Gamma_{E}}(\mathbf{y})\right\} \cdot\left\{\mathbf{n}_{\mathbf{x}} \times \nabla_{\mathbf{x}} \delta \psi_{\Gamma_{E}}(\mathbf{x})\right\} d s_{\mathbf{y}} d s_{\mathbf{x}},
\end{gathered}
$$

où $\langle.,$.$\rangle designe classiquement le crochet de dualité entre l'espace des fonctions admissibles sur \Gamma_{E}$ et son dual et où $G(\mathbf{x}-\mathbf{y})$ est la fonction de Green du problème qui s'écrit $G(\mathbf{x}-\mathbf{y})=g(\|\mathbf{x}-\mathbf{y}\|)$ avec $g(\|\mathbf{x}-\mathbf{y}\|)=-(4 \pi)^{-1} e^{-i k\|\mathbf{x}-\mathbf{y}\|} / r$. L'opérateur $\mathbf{H}\left(\omega / c_{E}\right)$ apparaissant dans le membre de droite de l'Eq. [8] a la propriété de symétrie ${ }^{t} \mathbf{H}\left(\omega / c_{E}\right)=\mathbf{H}\left(\omega / c_{E}\right)$. L'élimination de $\psi_{\Gamma_{E}}$ dans l'Eq. [8] donne une équation linéaire entre $\psi_{\Gamma_{E}}^{\text {sol }}$ et $v$ qui déf nit l'opérateur $\mathbf{B}_{\Gamma_{E}}\left(\omega / c_{E}\right)$. La procédure d'élimination de $\psi_{\Gamma_{E}}$ est expliquée dans le paragraphe 4.5 relatif au problème discrétisé. Cette procédure peut être effectuée pour toute valeur réelle de $\omega$. Il est à noter que la formulation proposée, d'une part conduit à un problème numérique avec matrice symétrique et, d'autre part ne présente pas le problème (dit des fréquences irrégulières) rencontré dans les formulations par équations intégrales (mauvaise construction de l'opérateur $\mathbf{B}_{\Gamma_{E}}\left(\omega / c_{E}\right)$ pour un ensemble dénombrable de valeurs de $\omega$ ). Concernant le problème des fréquences irrégulières, on renvoie le lecteur à [PAN 65] [SCH 68] [BUR 71] [ANG 83] [AMI 90] [COL 92] [OHA 98].

\subsection{Construction de l'opérateur de rayonnement acoustique}

Pour tout $\mathbf{x}$ dans $\Omega_{E}$, on a la représentation intégrale

$$
\psi^{\mathrm{sol}}(\mathbf{x}, \omega)=\int_{\Gamma_{E}}\left\{G(\mathbf{x}-\mathbf{y}) v(\mathbf{y})-\psi_{\Gamma_{E}}^{\mathrm{sol}}(\mathbf{y}, \omega) \frac{\partial G(\mathbf{x}-\mathbf{y})}{\partial \mathbf{n}_{\mathbf{y}}}\right\} d s_{\mathbf{y}} .
$$

En introduisant les opérateurs intégraux $\mathbf{R}_{\mathrm{S}}\left(\mathbf{x}, \omega / c_{E}\right)$ et $\mathbf{R}_{\mathrm{D}}\left(\mathbf{x}, \omega / c_{E}\right)$ déf nis par

$$
\begin{aligned}
\mathbf{R}_{\mathrm{S}}\left(\mathbf{x}, \omega / c_{E}\right) v & =\int_{\Gamma_{E}} G(\mathbf{x}-\mathbf{y}) v(\mathbf{y}) d s_{\mathbf{y}} \\
\mathbf{R}_{\mathrm{D}}\left(\mathbf{x}, \omega / c_{E}\right) \psi_{\Gamma_{E}} & =\int_{\Gamma_{E}} \psi_{\Gamma_{E}}(\mathbf{y}) \frac{\partial G(\mathbf{x}-\mathbf{y})}{\partial \mathbf{n}_{\mathbf{y}}} d s_{\mathbf{y}},
\end{aligned}
$$


l'Eq. [12] peut être réécrite $\psi^{\text {sol }}(\mathbf{x}, \omega)=\mathbf{R}\left(\mathbf{x}, \omega / c_{E}\right) v$ avec pour tout $\mathbf{x}$ dans $\Omega_{E}, \mathbf{R}\left(\mathbf{x}, \omega / c_{E}\right)=\mathbf{R}_{\mathrm{S}}\left(\mathbf{x}, \omega / c_{E}\right)-$ $\mathbf{R}_{\mathrm{D}}\left(\mathbf{x}, \omega / c_{E}\right) \mathbf{B}_{\Gamma_{E}}\left(\omega / c_{E}\right)$. Dans ces conditions, l'opérateur d'impédance de rayonnement acoustique $\mathbf{Z}_{\mathrm{rad}}(\mathbf{x}, \omega)$ s'écrit

$$
\mathbf{Z}_{\mathrm{rad}}(\mathbf{x}, \omega)=-i \omega \rho_{E}\left\{\mathbf{R}_{\mathrm{S}}\left(\mathbf{x}, \omega / c_{E}\right)-\mathbf{R}_{\mathrm{D}}\left(\mathbf{x}, \omega / c_{E}\right) \mathbf{B}_{\Gamma_{E}}\left(\omega / c_{E}\right)\right\} .
$$

\subsection{Discrétisation des opérateurs intégraux par éléments finis : éléments finis de frontière}

On utilise la méthode des éléments f nis pour discrétiser les opérateurs intégraux $\mathbf{S}_{\mathrm{S}}\left(\omega / c_{E}\right), \mathbf{S}_{\mathrm{D}}\left(\omega / c_{E}\right)$ et $\mathbf{S}_{\mathrm{T}}\left(\omega / c_{E}\right)$ (méthode dite des éléments f nis de frontière [NED 76] [AMI 92] [ANG 92] [BRE 92] [CHE 92] [DAU 92] [HAC 95]). On considère un maillage éléments f nis de la surface $\Gamma_{E}$ et soient $\mathbf{V}=\left(V_{1}, \ldots, V_{n_{E}}\right)$ et $\Psi_{\Gamma_{E}}=\left(\Psi_{\Gamma_{E}}, 1, \ldots, \Psi_{\Gamma_{E}}, n_{E}\right)$ les vecteurs complexes des $n_{E}$ DDL constitués par les valeurs de $v$ et $\psi_{\Gamma_{E}}$ aux $n_{p}$ noeuds du maillage. Soient $\left[S_{\mathrm{S}}\left(\omega / c_{E}\right)\right],\left[S_{\mathrm{D}}\left(\omega / c_{E}\right)\right]$ et $\left[S_{\mathrm{T}}\left(\omega / c_{E}\right)\right]$ les matrices complexes pleines $\left(n_{E} \times n_{E}\right)$ de la discrétisation des formes bilinéaires déf nies par les Eqs. [9] à [11]. Les matrices complexes $\left[S_{\mathrm{S}}\left(\omega / c_{E}\right)\right]$ et $\left[S_{\mathrm{T}}\left(\omega / c_{E}\right)\right]$ sont symétriques. Dans ces conditions, la discrétisation par éléments $\mathrm{f}$ nis de l'Eq. [8] s'écrit

$$
\left[\begin{array}{c}
\mathbf{0} \\
\boldsymbol{\Psi}_{\Gamma_{E}}^{\text {sol }}
\end{array}\right]=\left[\begin{array}{cc}
-\left[S_{\mathrm{T}}\left(\omega / c_{E}\right)\right] & \frac{1}{2}[E]^{T}-\left[S_{\mathrm{D}}\left(\omega / c_{E}\right)\right]^{T} \\
\frac{1}{2}[E]-\left[S_{\mathrm{D}}\left(\omega / c_{E}\right)\right] & {\left[S_{\mathrm{S}}\left(\omega / c_{E}\right)\right]}
\end{array}\right]\left[\begin{array}{l}
\boldsymbol{\Psi}_{\Gamma_{E}} \\
\mathbf{V}
\end{array}\right]
$$

avec $\Psi_{\Gamma_{E}}^{\text {sol }}$ le vecteur complexe des coordonnées généralisées qui correspondent à la discrétisation par éléments f nis de la forme linéaire $\delta v \mapsto \int_{\Gamma_{E}} \psi_{\Gamma_{E}}^{\text {sol }}(\mathbf{y}) \delta v(\mathbf{y}) d s_{\mathbf{y}}$. La matrice $[E]$ est la matrice réelle non-diagonale $\left(n_{E} \times n_{E}\right)$ qui correspond à la discrétisation de l'opérateur identité $\mathbf{I}$. L'élimination de $\boldsymbol{\Psi}_{\Gamma_{E}}$ dans l'Eq. [15] donne une équation linéaire entre $\Psi_{\Gamma_{E}}^{\text {sol }}$ et $\mathbf{V}$ qui déf nit la matrice complexe symétrique $\left[B_{\Gamma_{E}}\left(\omega / c_{E}\right)\right]$ de dimension $\left(n_{E} \times n_{E}\right)$ qui représente la discrétisation de l'opérateur $\mathbf{B}_{\Gamma_{E}}\left(\omega / c_{E}\right)$. On a

$$
\Psi_{\Gamma_{E}}^{\mathrm{sol}}=\left[B_{\Gamma_{E}}\left(\omega / c_{E}\right)\right] \mathbf{V}
$$

La procédure d'élimination est la suivante. Le vecteur $\Psi_{\Gamma_{E}}$ est éliminé en utilisant l'algorithme d'élimination de Gauss avec pivotage des lignes. En un $\omega$ donné, si la matrice $\left[S_{\mathrm{T}}\left(\omega / c_{E}\right)\right]$ est régulière, alors l'élimination peut être effectuée jusqu'à la ligne $n_{E}$. Pour un $\omega$, si la matrice $\left[S_{\mathrm{T}}\left(\omega / c_{E}\right)\right]$ est singulière, on note $n_{\alpha}<n_{E}$ la dimension du noyau qui est inconnue de la matrice $\left[S_{\mathrm{T}}\left(\omega / c_{E}\right)\right]$ (noyau qui est réel). Dans ce cas, l'élimination avec pivotage des lignes est arrêtée dès que l'on rencontre le premier pivot nul (zéro numérique relatif). L'élimination a donc été effectuée jusqu'à la ligne $n_{E}-n_{\alpha}$. Les équations qui correspondent aux lignes $n_{E}-n_{\alpha}+1, \ldots, n_{E}$ sont alors automatiquement satisfaites (résultat prouvé théoriquement et constaté numériquement). On construit ainsi pour tout $\omega$ réel la matrice symétrique complexe $\left[B_{\Gamma_{E}}\left(\omega / c_{E}\right)\right]$ (sans problèmes de fréquences irrégulières). On déduit de l'Eq. [7] l'expression de la matrice complexe symétrique $\left[Z_{\Gamma_{E}}(\omega)\right]=-i \omega \rho_{E}\left[B_{\Gamma_{E}}\left(\omega / c_{E}\right)\right]$ de dimension $\left(n_{E} \times n_{E}\right)$ qui discrétise l'opérateur de frontière d'impédance acoustique $\mathbf{Z}_{\Gamma_{E}}(\omega)$. Finalement, la discrétisation correspondante de l'opérateur d'impédance de rayonnement acoustique $\mathbf{Z}_{\mathrm{rad}}(\mathbf{x}, \omega)$ déf ni par l'Eq. [14] s'écrit

$$
\left[Z_{\mathrm{rad}}(\mathbf{x}, \omega)\right]=-i \omega \rho_{E}\left\{\left[R_{\mathrm{S}}\left(\mathbf{x}, \omega / c_{E}\right)\right]-\left[R_{\mathrm{D}}\left(\mathbf{x}, \omega / c_{E}\right)\right]\left[B_{\Gamma_{E}}\left(\omega / c_{E}\right)\right]\right\} .
$$

\subsection{Réponse acoustique pour un champ de déplacement de paroi, une densité de source acoustique et une onde plane incidente donnés}

Considérons les excitations acoustiques externes décrites au paragraphe 3 et supposons que le champ de vitesse normale sur $\Gamma_{E}$ soit donné par $v=i \omega \mathbf{u}(\omega) \cdot \mathbf{n}^{S}$ où $\mathbf{n}^{S}$ désigne la normale unitaire extérieure à $\Omega_{S}$. Alors, en utilisant la linéarité du problème, le champ de pression $\left.p_{E}\right|_{\Gamma_{E}}(\omega)$ sur la paroi $\Gamma_{E}$ s'écrit

$$
\left.p_{E}\right|_{\Gamma_{E}}(\omega)=i \omega \mathbf{Z}_{\Gamma_{E}}(\omega)\left\{\mathbf{u}(\omega) \cdot \mathbf{n}^{S}\right\}+\left.p_{\text {donnée }}\right|_{\Gamma_{E}}(\omega),
$$

où $\mathbf{Z}_{\Gamma_{E}}(\omega)$ est l'opérateur de frontière d'impédance acoustique. Le champ de pression $p_{E}(\mathbf{x}, \omega)$ dans le f uide extérieur $\Omega_{E}$ s'écrit

$$
p_{E}(\mathbf{x}, \omega)=i \omega \mathbf{Z}_{\mathrm{rad}}(\mathbf{x}, \omega)\left\{\mathbf{u}(\omega) \cdot \mathbf{n}^{S}\right\}+p_{\text {donnée }}(\mathbf{x}, \omega),
$$


où $\mathbf{Z}_{\mathrm{rad}}(\mathbf{x}, \omega)$ est l'opérateur d'impédance de rayonnement acoustique dans le f uide extérieur. Dans les Eqs. [18] et [19], le champ de pression $\left.p_{\text {donnée }}\right|_{\Gamma_{E}}(\omega)$ sur la paroi $\Gamma_{E}$ et le champ de pression $p_{\text {donnée }}(\mathbf{x}, \omega)$ dans $\Omega_{E}$ s'expriment en fonction des excitations acoustiques $Q(\mathbf{x}, \omega)$ et $\psi_{\text {inc }}(\mathbf{x}, \omega)$ et des opérateurs $\mathbf{Z}_{\Gamma_{E}}(\omega)$ et $\mathbf{Z}_{\mathrm{rad}}(\mathbf{x}, \omega)$ (voir chap. 12 de [OHA 98]).

\section{Modélisation, discrétisation par éléments finis et réduction modale en vibroacoustique basses fréquences}

On considère le problème décrit au paragraphe 3 (voir Figure 2). Concernant les détails des développements qui suivent, on renvoie le lecteur au chap. 13 de [OHA 98].

\subsection{Expression du champ de pression dans le fluide acoustique interne dissipatif}

Rappelons que dans le cas d'un fuide acoustique interne parfait (donc non dissipatif), si il existe une condition de type $p=0$ sur une partie du bord $\partial \Omega$, alors le champ de pression $p(\mathbf{x}, \omega)$ est relié au potentiel des déplacements $\widetilde{\varphi}(\mathbf{x}, \omega)$ par la relation classique

$$
p(\mathbf{x}, \omega)=\omega^{2} \rho_{0} \widetilde{\varphi}(\mathbf{x}, \omega)
$$

où $\rho_{0}$ est la masse volumique du f uide à l'équilibre. L'équation correspondante dans ce f uide parfait est alors l'équation d'Helmholtz en $\widetilde{\varphi}$ (ou en $p$ ). Pour ce même f uide parfait, s'il n'existe pas une condition de type $p=0$ sur une partie du bord $\partial \Omega$ (cas de la Figure 2), alors il a été démontré [OHA 87] [OHA 90] [MOR 95] (en particulier pour le calcul des modes élastoacoustiques et la construction de modèles réduits matriciels par sous-structuration dynamique), que l'Eq. [20] est fausse pour une paroi qui se déforme et que cette équation doit être remplacée par

$$
p(\mathbf{x}, \omega)=\omega^{2} \rho_{0} \widetilde{\varphi}(\mathbf{x}, \omega)+\pi(\omega ; \mathbf{u} ; \widetilde{\varphi}),
$$

où $\pi(\omega ; \mathbf{u} ; \widetilde{\varphi})$ est un champ constant dans $\Omega$, mais qui dépend de $\mathbf{u}$ et $\widetilde{\varphi}$.

Dans le présent papier (voir paragraphe 3), le f uide acoustique interne n'est pas parfait mais dissipatif et il y a des sources acoustiques internes ([OHA 98], chap. 10). Il existe un potentiel de déplacement $\widetilde{\varphi}$ déf ni à une constante additive près et on introduit un champ scalaire $\varphi$ relié au potentiel de déplacement $\widetilde{\varphi}$ par la relation [SOI 92] [OHA 98]

$$
\widetilde{\varphi}(\mathbf{x}, \omega)=(1+i \omega \tau) \varphi(\mathbf{x}, \omega)-\frac{\tau c_{0}^{2}}{\rho_{0}} \frac{Q(\mathbf{x}, \omega)}{\omega^{2}},
$$

où $c_{0}$ est la célérité du son à l'équilibre et où $\tau$ est un coeff cient qui ne dépend que de la viscosité dynamique $\eta$ et de la seconde viscosité $\zeta$ du fuide, qui peut dépendre de $\omega$ et qui s'écrit

$$
\tau=\frac{1}{\rho_{0} c_{0}^{2}}\left(\frac{4}{3} \eta+\zeta\right)>0
$$

Dans ces conditions, on montre [OHA 98] que 1'Eq. [21] doit être remplacée par

$$
p(\mathbf{x}, \omega)=\omega^{2} \rho_{0} \varphi(\mathbf{x}, \omega)+\pi(\omega ; \mathbf{u} ; \varphi),
$$

où le champ $\varphi$ doit être tel que

$$
\int_{\Omega} \varphi d \mathbf{x}=0
$$

et où $\pi(\omega ; \mathbf{u} ; \varphi)$ s'écrit

$$
\pi(\omega ; \mathbf{u} ; \varphi)=\kappa(\omega)\left\{-\omega^{2} \rho_{0} \pi_{1}(\omega ; \varphi)-\pi_{2}(\mathbf{u})+\pi_{\mathrm{Q}}(\omega)\right\}
$$

Dans l'Eq. [26], $\pi_{1}(\omega ; \varphi), \pi_{2}(\mathbf{u})$ et $\pi_{\mathrm{Q}}(\omega)$ sont donnés par

$$
\pi_{1}(\omega ; \varphi)=\int_{\Gamma_{Z}} \frac{\varphi(\mathbf{x}, \omega)}{i \omega Z(\mathbf{x}, \omega)} d s(\mathbf{x})
$$




$$
\begin{gathered}
\pi_{2}(\mathbf{u})=\int_{\Gamma \cup \Gamma_{Z}} \mathbf{u}(\mathbf{x}, \omega) \cdot \mathbf{n}(\mathbf{x}) d s(\mathbf{x}), \\
\pi_{\mathrm{Q}}(\omega)=\frac{1}{\rho_{0}} \int_{\Omega} \frac{Q(\mathbf{x}, \omega)}{i \omega} d \mathbf{x} .
\end{gathered}
$$

Dans l'Eq. [26], $\kappa(\omega)$ est un nombre complexe donné par

$$
\kappa(\omega)=\frac{\rho_{0} c_{0}^{2}}{|\Omega|} \times\left\{1+\frac{\rho_{0} c_{0}^{2}}{|\Omega|} \int_{\Gamma_{Z}} \frac{d s(\mathbf{x})}{i \omega Z(\mathbf{x}, \omega)}\right\}^{-1}
$$

où $|\Omega|$ désigne le volume du domaine $\Omega$.

\subsection{Problème aux limites pour le système vibroacoustique}

Equations pour la structure. L'équation d'équilibre dynamique pour la structure $\Omega_{S}$ s'écrit

$$
-\omega^{2} \rho_{S} u_{i}-\sigma_{i j, j}(\mathbf{u})=g_{i} \quad \text { dans } \quad \Omega_{S},
$$

où $\rho_{S}(\mathbf{x})$ est la masse volumique de la structure. Dans l'Eq. [31], la loi de comportement pour le domaine BF s'écrit (voir chap. 4 de [OHA 98])

$$
\sigma_{i j}(\mathbf{x}, \omega)=\left(a_{i j k h}(\mathbf{x})+i \omega b_{i j k h}(\mathbf{x}, \omega)\right) \varepsilon_{k h}(\mathbf{x}, \omega)
$$

où $\varepsilon_{k h}(\mathbf{x}, \omega)=\frac{1}{2}\left(u_{k, h}+u_{h, k}\right)$ est le tenseur de déformation linéarisé. Les constantes $a_{i j k h}$ sont indépendantes de $\omega$ pour la bande BF (ce qui ne sera pas le cas pour la bande MF). La condition aux limites sur $\Gamma_{E}$ s'écrit $\sigma_{i j}(\mathbf{u}) n_{j}^{S}=G_{i}-\left.p_{E}\right|_{\Gamma_{E}} n_{i}^{S}$, soit en utilisant l'Eq. [18],

$$
\sigma_{i j}(\mathbf{u}) n_{j}^{S}=G_{i}-\left.p_{\text {donnée }}\right|_{\Gamma_{E}} n_{i}^{S}-i \omega \mathbf{Z}_{\Gamma_{E}}(\omega)\left\{\mathbf{u} \cdot \mathbf{n}^{S}\right\} n_{i}^{S} \quad \text { sur } \quad \Gamma_{E} .
$$

La condition aux limites sur $\Gamma \cup \Gamma_{Z}$ s'écrit $\sigma_{i j}(\mathbf{u}) n_{j}^{S}=G_{i}-p(\omega) n_{i}^{S}=G_{i}+p(\omega) n_{i}$ où $\mathbf{n}=-\mathbf{n}^{S}$, soit en utilisant les Eqs. [24] et [26],

$$
\sigma_{i j}(\mathbf{u}) n_{j}^{S}=G_{i}+\kappa \pi_{\mathrm{Q}} n_{i}+\omega^{2} \rho_{0} \varphi n_{i}-\omega^{2} \rho_{0} \kappa \pi_{1}(\omega ; \varphi) n_{i}-\kappa \pi_{2}(\mathbf{u}) n_{i} \quad \text { sur } \quad \Gamma \cup \Gamma_{Z} .
$$

Equations pour le fluide acoustique interne dissipatif. Concernant le f uide interne, 1'Eq. [24] peut s'écrire

$$
p(\mathbf{x}, \omega)=p^{\prime}(\mathbf{x}, \omega)+\pi(\omega ; \mathbf{u} ; \varphi)
$$

avec

$$
p^{\prime}(\mathbf{x}, \omega)=\omega^{2} \rho_{0} \varphi(\mathbf{x}, \omega)
$$

que l'on peut réécrire

$$
\frac{1}{\rho_{0} c_{0}^{2}} p^{\prime}=\frac{\omega^{2}}{c_{0}^{2}} \varphi \quad \text { dans } \quad \Omega .
$$

Les champs $p^{\prime}$ et $\varphi$ doivent satisfaire les contraintes

$$
\begin{aligned}
& \int_{\Omega} p^{\prime} d \mathbf{x}=0 \\
& \int_{\Omega} \varphi d \mathbf{x}=0
\end{aligned}
$$

Il a été démontré (voir [MOR 95] pour le cas spectral non dissipatif et [OHA 98] pour le cas dissipatif avec sources et impédances de parois) que, pour obtenir des formulations symétriques pour le couplage de la structure avec le fuide interne, permettant le calcul des modes élastoacoustiques par résolution de problèmes généralisés aux valeurs propres du type $[A] \mathbf{X}=\omega^{2}[B] \mathbf{X}$ avec $[A]$ et $[B]$ des matrices symétriques réelles (à l'aide de modèles éléments $\mathrm{f}$ nis avec matrices réelles symétriques conduisant à des modèles modaux réduits 
avec matrices réelles symétriques), il fallait introduire simultanément pour le fuide deux champs inconnus scalaires liés au champ de pression et au potentiel de déplacement. L'équation régissant la dynamique du fuide acoustique interne dissipatif s'écrit alors en terme des champs scalaires $p^{\prime}$ et $\varphi$,

$$
-\frac{1}{c_{0}^{2}} p^{\prime}-i \omega \tau \rho_{0} \nabla^{2} \varphi-\rho_{0} \nabla^{2} \varphi=g_{\mathrm{Q}}-\frac{\omega^{2} \rho_{0} \kappa}{c_{0}^{2}} \pi_{1}(\omega ; \varphi)-\frac{1}{c_{0}^{2}} \kappa \pi_{2}(\mathbf{u}) \text { dans } \Omega,
$$

où $g_{\mathrm{Q}}$ est une fonction donnée, liée à la source acoustique interne $\mathbf{Q}$, qui est déf nie par l'Eq. [44] ci-après. La condition de Neumann sur $\Gamma$ s'écrit

$$
\rho_{o}(1+i \omega \tau) \frac{\partial \varphi}{\partial \mathbf{n}}=\rho_{0} \mathbf{u} \cdot \mathbf{n}+G_{\mathrm{Q}} \quad \text { sur } \quad \Gamma,
$$

où $G_{\mathrm{Q}}$ est une fonction donnée, liée à la source acoustique interne $\mathbf{Q}$, qui est déf nie par l'Eq. [45] ci-après. La frontière $\Gamma_{Z}$ (voir Figure 2) a des propriétés acoustiques modélisées par une impédance acoustique de paroi $Z(\mathbf{x}, \omega)$ à valeurs complexes qui est telle que

$$
p(\mathbf{x}, \omega)=Z(\mathbf{x}, \omega)\{\mathbf{v}(\mathbf{x}, \omega) \cdot \mathbf{n}(\mathbf{x})-i \omega \mathbf{u}(\mathbf{x}, \omega) \cdot \mathbf{n}(\mathbf{x})\}, \forall \mathbf{x} \in \Gamma_{Z},
$$

où $\mathbf{v}(\mathbf{x}, \omega)$ est la vitesse dans le fuide interne qui s'écrit $\mathbf{v}(\mathbf{x}, \omega)=i \omega \nabla \widetilde{\varphi}(\mathbf{x}, \omega)$ avec $\widetilde{\varphi}(\mathbf{x}, \omega)$ donné par l'Eq. [22] et où $\mathbf{n}=-\mathbf{n}^{S}$. La condition de Neumann sur $\Gamma_{Z}$ s'écrit alors

$$
\rho_{0}(1+i \omega \tau) \frac{\partial \varphi}{\partial \mathbf{n}}=\rho_{0} \mathbf{u} \cdot \mathbf{n}+\frac{\omega^{2} \rho_{0}^{2}}{i \omega Z} \varphi-\frac{\omega^{2} \rho_{0}^{2} \kappa}{i \omega Z} \pi_{1}(\omega ; \varphi)-\frac{\kappa \rho_{0}}{i \omega Z} \pi_{2}(\mathbf{u})+G_{\mathrm{Q}}+G_{\mathrm{Q}, \mathrm{Z}}
$$

où $G_{\mathrm{Q}, \mathrm{Z}}$ est une fonction donnée, liée à la source acoustique interne $\mathbf{Q}$, qui est déf nie par l'Eq. [46] ci-après. La fonction $g_{\mathrm{Q}}$ est déf nie dans $\Omega$ par

$$
g_{\mathrm{Q}}(\mathbf{x}, \omega)=\frac{1}{c_{0}^{2}} \kappa(\omega) \pi_{\mathrm{Q}}(\omega)-\frac{1}{i \omega} Q(\mathbf{x}, \omega)-\tau c_{0}^{2} \frac{1}{\omega^{2}} \nabla^{2} Q(\mathbf{x}, \omega)
$$

$G_{\mathrm{Q}}$ est déf nie sur $\Gamma \cup \Gamma_{Z}$ par

$$
G_{\mathrm{Q}}(\mathbf{x}, \omega)=\tau c_{0}^{2} \frac{1}{\omega^{2}} \frac{\partial Q(\mathbf{x}, \omega)}{\partial \mathbf{n}(\mathbf{x})}
$$

et $G_{\mathrm{Q}, Z}$ est déf nie sur $\Gamma_{Z}$ par

$$
G_{\mathrm{Q}, Z}(\mathbf{x}, \omega)=\frac{\rho_{0}}{i \omega Z(\mathbf{x}, \omega)} \kappa(\omega) \pi_{\mathrm{Q}}(\omega)
$$

\subsection{Discrétisation par éléments finis}

On considère un maillage éléments f nis de la structure $\Omega_{S}$ et du fuide interne $\Omega$, les deux maillages étant supposés compatibles sur l'interface $\Gamma \cup \Gamma_{Z}$. Soit $\mathbf{U}=\left(U_{1}, \ldots, U_{n_{S}}\right)$ le vecteur complexe des $n_{S}$ DDL de la structure qui sont les valeurs nodales du champ de déplacement $\mathbf{u}$. Soient $\boldsymbol{\Phi}=\left(\Phi_{1}, \ldots, \Phi_{n}\right)$ et $\mathbf{P}^{\prime}=\left(p_{1}^{\prime}, \ldots, p_{n}^{\prime}\right)$ les vecteurs complexes des $n$ DDL du fuide qui sont les valeurs nodales des champs $\varphi$ et $p^{\prime}$. La discrétisation par éléments $\mathrm{f}$ nis de la formulation variationnelle en $\mathbf{u}, \varphi$ et $p^{\prime}$ du problème aux limites déf ni par les Eqs. [31] à [46], conduit au système complexe symétrique suivant

$$
[A(\omega)]\left[\begin{array}{c}
\mathbf{U} \\
\mathbf{\Phi} \\
\mathbf{P}^{\prime}
\end{array}\right]=\left[\begin{array}{c}
\mathbf{F}_{\mathrm{S}}(\omega) \\
\omega^{2} \mathbf{F}_{\mathrm{Q}}(\omega) \\
\mathbf{0}
\end{array}\right],
$$

avec les contraintes correspondant à la discrétisation par éléments f nis des Eqs. [38] et [39]

$$
\mathbf{L}^{T} \mathbf{P}^{\prime}=0 \quad, \quad \mathbf{L}^{T} \boldsymbol{\Phi}=0
$$


et où $\mathbf{L}$ est un vecteur de $\mathbb{R}^{n}$. La matrice complexe symétrique $[A(\omega)]$ est déf nie par

$$
\left[\begin{array}{ccc}
{\left[A^{S}(\omega)\right]-\omega^{2}\left[A^{E}\left(\omega / c_{E}\right)\right]+\kappa(\omega)[J]} & -\omega^{2}[C(\omega)] & {[0]} \\
-\omega^{2}[C(\omega)]^{T} & i \omega^{3}\left[D_{\tau}(\omega)\right]+\omega^{2}[K]+\omega^{2}\left[S_{Z}(\omega)\right] & -\frac{\omega^{2}}{c_{0}^{2}}\left[C_{1}\right] \\
{[0]} & -\frac{\omega^{2}}{c_{0}^{2}}\left[C_{1}\right]^{T} & \frac{1}{\rho_{0}^{2}}[M]
\end{array}\right]
$$

où $\left[A^{S}(\omega)\right]$ est la matrice complexe symétrique $\left(n_{S} \times n_{S}\right)$ de raideur dynamique de la structure (qui n'est singulière que pour $\omega=0$ de par la présence des modes de corps rigide) qui s'écrit

$$
\left[A^{S}(\omega)\right]=-\omega^{2}\left[M^{S}\right]+i \omega\left[D^{S}(\omega)\right]+\left[K^{S}\right]
$$

avec $\left[M^{S}\right],\left[D^{S}(\omega)\right]$ et $\left[K^{S}\right]$ les matrices $\left(n_{S} \times n_{S}\right)$ réelles symétriques de masse, de dissipation et de raideur de la structure in vacuo, la matrice $\left[M^{S}\right]$ étant déf nie positive et les matrices $\left[D^{S}(\omega)\right]$ et $\left[K^{S}\right]$ étant positives (et ayant le même noyau). La matrice $\left(n_{S} \times n_{S}\right)$ complexe symétrique $\left[A^{E}\left(\omega / c_{E}\right)\right]$ qui traduit les effets du f uide extérieur sur la structure, s'écrit

$$
\left[A^{E}\left(\omega / c_{E}\right)\right]=-\rho_{E}[\Theta]^{T}\left[B_{\Gamma_{E}}\left(\omega / c_{E}\right)\right][\Theta],
$$

où $\left[B_{\Gamma_{E}}\left(\omega / c_{E}\right)\right]$ est la matrice $\left(n_{E} \times n_{E}\right)$ pleine complexe symétrique déf nie au paragraphe 4.5 et où la matrice creuse rectangulaire $\left(n_{E} \times n_{S}\right)$ réelle $[\Theta]$ correspond à la discrétisation $\overline{\delta \mathbf{W}}^{T}[\Theta] \mathbf{U}$ par éléments f nis de la forme sesquilinéaire $(\mathbf{u}, \delta w) \mapsto \theta(\mathbf{u}, \delta w)=\int_{\Gamma_{E}} \overline{\delta w} \mathbf{u} \cdot \mathbf{n}^{S} d s$ qui projette $\mathbf{u}$ sur la normale à la surface $\Gamma_{E}$. Il est à noter que la matrice $\left[A^{E}\left(\omega / c_{E}\right)\right]$ a des lignes et des colonnes de zéros pour les $n_{p}$ noeuds qui ne sont pas localisés sur $\Gamma_{E}$. La matrice $[J]$ est une matrice $\left(n_{S} \times n_{S}\right)$ réelle symétrique qui s'écrit

$$
[J]=\Pi_{2} \Pi_{2}^{T},
$$

où $\Pi_{2}$ est le vecteur dans $\mathbb{R}^{n_{S}}$ tel que $\Pi_{2}^{T}$ correspond à la discrétisation de la forme linéaire $\pi_{2}(\mathbf{u})$ déf nie par l'Eq. [28]. La matrice [ $J]$ a des lignes et des colonnes de zéros pour les $n_{p}$ noeuds qui ne sont pas localisés sur $\Gamma \cup \Gamma_{Z}$. La matrice $(n \times n)$ réelle symétrique positive $[K]$ correspond à la discrétisation de la forme sesquilinéaire $k(\varphi, \delta \varphi)=\rho_{0} \int_{\Omega} \boldsymbol{\nabla} \varphi \cdot \nabla \overline{\delta \varphi} d \mathbf{x}$. La matrice $\left[D_{\tau}(\omega)\right.$ est déf nie par

$$
\left[D_{\tau}(\omega)\right]=\tau(\omega)[K],
$$

La matrice $(n \times n)$ complexe symétrique $\left[S_{Z}(\omega)\right]$ correspond à la discrétisation de la forme sesquilinéaire $s_{Z}(\omega ; \varphi, \delta \varphi)=-\rho_{0}^{2} \omega^{2} \int_{\Gamma_{Z}}(i \omega Z)^{-1} \varphi \overline{\delta \varphi} d s+\omega^{2} \kappa(\omega) \rho_{0}^{2} \pi_{1}(\omega ; \varphi) \pi_{1}(\omega ; \overline{\delta \varphi})$ et est telle que $\omega^{-1} \Im m\left[S_{Z}(\omega)\right]$ est positive. Il est à noter que la matrice $\left[S_{Z}(\omega)\right]$ a des lignes et des colonnes de zéros pour les $n_{p}$ noeuds qui ne sont pas localisés sur $\Gamma_{Z}$. La matrice $(n \times n)$ réelle $[M]$ symétrique déf nie positive correspond à la discrétisation de la forme sesquilinéaire $m\left(p^{\prime}, \delta p^{\prime}\right)=\left(\rho_{0} / c_{0}^{2}\right) \int_{\Omega} p^{\prime} \overline{\delta p^{\prime}} d \mathbf{x}$. La matrice rectangulaire $\left(n_{S} \times n\right)$ complexe $[C(\omega)]$ s'écrit

$$
[C(\omega)]=\left[C_{0}\right]-\rho_{0} \kappa(\omega) \boldsymbol{\Pi}_{2} \boldsymbol{\Pi}_{1}(\omega)^{T},
$$

où la matrice $\left(n_{S} \times n\right)$ réelle $\left[C_{0}\right]$ correspond à la discrétisation de la forme sesquilinéaire $c_{0}(\varphi, \delta \mathbf{u})=$ $\rho_{0} \int_{\Gamma \cup \Gamma_{Z}} \varphi \mathbf{n} \cdot \overline{\delta \mathbf{u}} d s$ et où $\boldsymbol{\Pi}_{1}(\omega)$ est le vecteur dans $\mathbb{C}^{n}$ tel que $\boldsymbol{\Pi}_{1}(\omega)^{T}$ correspond à la discrétisation de la forme linéaire $\pi_{1}(\omega ; \varphi)$ déf nie par l'Eq. [27]. La matrice $[C(\omega)]$ a des lignes et des colonnes de zéros pour les $n_{p}$ noeuds qui ne sont pas localisés sur $\Gamma \cup \Gamma_{Z}$. La matrice $(n \times n)$ réelle symétrique $\left[C_{1}\right]$ s'écrit

$$
\left[C_{1}\right]=\frac{c_{0}^{2}}{\rho_{0}}[M]
$$

et correspond à la discrétisation de la forme sesquilinéaire $c_{1}\left(p^{\prime}, \delta \varphi\right)=\int_{\Omega} p^{\prime} \overline{\delta \varphi} d \mathbf{x}$. Le lecteur trouvera les expressions (en fonction des données) du second membre de l'Eq. [47] dans les chap. 11 et 13 de [OHA 98]. Soit $\mathbf{P}=\left(p_{1}, \ldots, p_{n}\right)$ le vecteur des DDL constitués des valeurs de la pression $p$ aux $n_{p}$ noeuds du maillage du f uide interne $\Omega$. Il est calculé par

$$
\mathbf{P}=\omega^{2} \rho_{0} \boldsymbol{\Phi}+\kappa(\omega)\left\{-\omega^{2} \rho_{0} \boldsymbol{\Pi}_{1}(\omega)^{T} \boldsymbol{\Phi}-\boldsymbol{\Pi}_{2}^{T} \mathbf{U}+\pi_{\mathrm{Q}}(\omega)\right\} \frac{\rho_{0}}{c_{0}^{2}}[M]^{-1} \mathbf{L} .
$$




\subsection{Modèle réduit matriciel symétrique}

On commence par calculer les modes de la structure in vacuo et les modes acoustiques de la cavité interne avec parois $\mathrm{f}$ xes. Les modes de la structure sont obtenus en résolvant le problème généralisé aux valeurs propres

$$
\left[K^{S}\right] \mathbf{U}=\lambda^{S}\left[M^{S}\right] \mathbf{U},
$$

qui donne les valeurs propres $\lambda_{\alpha}^{S}=\left\{\omega_{\alpha}^{S}\right\}^{2}$ telles que $0=\lambda_{1}^{S}=\ldots=\lambda_{6}^{S}<\lambda_{7}^{S} \leq \lambda_{8}^{S} \ldots$. Les vecteurs propres $\mathbf{U}_{1}, \ldots, \mathbf{U}_{6}$ associés aux valeurs propres $\lambda_{\alpha}^{S}=0$ sont les six modes de corps rigide et les vecteurs propres $\left\{\mathbf{U}_{\alpha}, \alpha \geq 7\right\}$ sont les modes élastiques. On a les propriétés d'orthogonalité pour $\alpha \geq 1$ et $\beta \geq 1$,

$$
\mathbf{U}_{\beta}^{T}\left[M^{S}\right] \mathbf{U}_{\alpha}=\mu_{\alpha}^{S} \delta_{\alpha \beta} \quad, \quad \mathbf{U}_{\beta}^{T}\left[K^{S}\right] \mathbf{U}_{\alpha}=\mu_{\alpha}^{S} \lambda_{\alpha}^{S} \delta_{\alpha \beta} \quad .
$$

Les modes acoustiques de la cavité avec parois $\mathrm{f}$ xes sont obtenus en résolvant le problème généralisé aux valeurs propres

$$
[K] \boldsymbol{\Phi}=\lambda[M] \boldsymbol{\Phi} \quad \text { avec } \quad \mathbf{L}^{T} \boldsymbol{\Phi}=0 \quad,
$$

qui donne les valeurs propres $\lambda_{\alpha}=\left\{\omega_{\alpha}\right\}^{2}$ telles que $0<\lambda_{1} \leq \lambda_{2} \ldots$. Les vecteurs propres $\left\{\boldsymbol{\Phi}_{1}, \boldsymbol{\Phi}_{2}, \ldots\right\}$ sont les modes acoustiques. On a les propriétés d'orthogonalité

$$
\boldsymbol{\Phi}_{\beta}^{T}[M] \mathbf{\Phi}_{\alpha}=\mu_{\alpha} \delta_{\alpha \beta} \quad, \quad \boldsymbol{\Phi}_{\beta}^{T}[K] \boldsymbol{\Phi}_{\alpha}=\mu_{\alpha} \lambda_{\alpha} \delta_{\alpha \beta} .
$$

Pratiquement, on résoud l'Eq. [59] sans la liaison et l'on ne conserve que les vecteurs propres associés aux valeurs propres strictement positives (modes acoustiques). Le modèle matriciel réduit est obtenu en projetant l'Eq. [47] sur les $N_{S}$ premiers modes de structure $\mathbf{U}_{1}, \ldots, \mathbf{U}_{N_{S}}$ (comprenant les modes rigides) et les $N$ premiers modes acoustiques $\boldsymbol{\Phi}_{1}, \ldots, \boldsymbol{\Phi}_{N}$. On introduit la matrice $\left(n_{S} \times N_{S}\right)$ réelle $[U]$ et la matrice $(n \times N)$ réelle $[\Phi]$ telles que

$$
[U]=\left[\mathbf{U}_{1} \ldots \mathbf{U}_{\alpha} \ldots \mathbf{U}_{N_{S}}\right] \quad, \quad[\Phi]=\left[\boldsymbol{\Phi}_{1} \ldots \Phi_{\alpha} \ldots \Phi_{N}\right] .
$$

On considère alors les changements de base

$$
\mathbf{U}=[U] \mathbf{q}^{S} \quad, \quad \boldsymbol{\Phi}=[\Phi] \mathbf{q} \quad, \quad \mathbf{P}^{\prime}=[\Phi] \mathbf{r} .
$$

La projection de l'Eq. [47] donne

$$
[\mathcal{A}(\omega)]\left[\begin{array}{c}
\mathbf{q}^{S} \\
\mathbf{q} \\
\mathbf{r}
\end{array}\right]=\left[\begin{array}{c}
\mathcal{F}_{\mathrm{S}}(\omega) \\
\omega^{2} \mathcal{F}_{\mathrm{Q}}(\omega) \\
\mathbf{0}
\end{array}\right],
$$

où la matrice complexe $[\mathcal{A}(\omega)]$ est symétrique et déf nie par

$$
\left[\begin{array}{ccc}
{\left[\mathcal{A}^{S}(\omega)\right]-\omega^{2}\left[\mathcal{A}^{E}\left(\omega / c_{E}\right)\right]+\kappa(\omega)[\mathcal{J}]} & -\omega^{2}[\mathcal{C}(\omega)] & {[0]} \\
-\omega^{2}[\mathcal{C}(\omega)]^{T} & i \omega^{3}\left[\mathcal{D}_{\tau}(\omega)\right]+\omega^{2}[\mathcal{K}]+\omega^{2}\left[\mathcal{S}_{z}(\omega)\right] & -\frac{\omega^{2}}{c_{0}^{2}}\left[\mathcal{C}_{1}\right] \\
{[0]} & -\frac{\omega^{2}}{c_{0}^{2}}\left[\mathcal{C}_{1}\right]^{T} & \frac{1}{\rho_{0}^{2}}[\mathcal{M}]
\end{array}\right]
$$

La matrice $\left[\mathcal{A}^{S}(\omega)\right]$ est une matrice complexe $\left(N_{S} \times N_{S}\right)$ symétrique qui s'écrit

$$
\left[\mathcal{A}^{S}(\omega)\right]=-\omega^{2}\left[\mathcal{M}^{S}\right]+i \omega\left[\mathcal{D}^{S}(\omega)\right]+\left[\mathcal{K}^{S}\right],
$$

avec $\left[\mathcal{M}^{S}\right]$ la matrice de masse généralisée de la structure qui est réelle, $\left(N_{S} \times N_{S}\right)$, diagonale, déf nie positive, $\left[\mathcal{D}^{S}(\omega)\right]$ la matrice généralisée de dissipation de la structure qui est réelle, $\left(N_{S} \times N_{S}\right)$, pleine, symétrique, positive et $\left[\mathcal{K}^{S}\right]$ la matrice de raideur généralisée qui est une matrice réelle, $\left(N_{S} \times N_{S}\right)$, diagonale, positive, telles que

$$
\begin{aligned}
{\left[\mathcal{M}^{S}\right]_{\alpha \beta} } & =\mu_{\alpha}^{S} \delta_{\alpha \beta} \quad, \quad\left[\mathcal{D}^{S}(\omega)\right]=[U]^{T}\left[D^{S}(\omega)\right][U] \\
{\left[\mathcal{K}^{S}\right]_{\alpha \beta} } & =\mu_{\alpha}^{S} \lambda_{\alpha}^{S} \delta_{\alpha \beta}
\end{aligned}
$$


La matrice $\left(N_{S} \times N_{S}\right)$ complexe symétrique $\left[\mathcal{A}^{E}\left(\omega / c_{E}\right)\right]$ s'écrit

$$
\left[\mathcal{A}^{E}\left(\omega / c_{E}\right)\right]=-\rho_{E}\left[U_{\Gamma_{E}}\right]^{T}\left[B_{\Gamma_{E}}\left(\omega / c_{E}\right)\right]\left[U_{\Gamma_{E}}\right] \quad \text { avec } \quad\left[U_{\Gamma_{E}}\right]=[\Theta][U] .
$$

La matrice $[\mathcal{J}]$ est une matrice réelle, $\left(N_{S} \times N_{S}\right)$, symétrique, positive, qui s'écrit

$$
[\mathcal{J}]=\left(\boldsymbol{\Pi}_{2}^{T}[U]\right)^{T}\left(\boldsymbol{\Pi}_{2}^{T}[U]\right) .
$$

Les matrices $[\mathcal{M}],[\mathcal{K}]$ et $\left[\mathcal{D}_{\tau}(\omega)\right]$ sont des matrices réelles, $(N \times N)$, diagonales, déf nies positives, telles que

$$
[\mathcal{M}]_{\alpha \beta}=\mu_{\alpha} \delta_{\alpha \beta} \quad, \quad[\mathcal{K}]_{\alpha \beta}=\mu_{\alpha} \lambda_{\alpha} \delta_{\alpha \beta} \quad, \quad\left[\mathcal{D}_{\tau}(\omega)\right]_{\alpha \beta}=\tau(\omega) \mu_{\alpha} \lambda_{\alpha} \delta_{\alpha \beta}
$$

La matrice $\left[\mathcal{S}_{Z}(\omega)\right]$ est une matrice complexe $(N \times N)$ pleine symétrique qui s'écrit $\left[\mathcal{S}_{Z}(\omega)\right]=[\Phi]^{T}\left[S_{Z}(\omega)\right][\Phi]$ et qui n'utilise que les valeurs des modes acoustiques sur la surface $\Gamma \cup \Gamma_{Z}$. La matrice $[\mathcal{C}(\omega)]$ est une matrice complexe, rectangulaire $\left(N_{S} \times N\right)$, pleine, qui s'écrit

$$
[\mathcal{C}(\omega)]=[U]^{T}\left[C_{0}(\omega)\right][\Phi]-\rho_{0} \kappa(\omega)\left(\boldsymbol{\Pi}_{2}^{T}[U]\right)^{T}\left(\boldsymbol{\Pi}_{1}(\omega)^{T}[\Phi]\right)
$$

La matrice $\left[\mathcal{C}_{1}\right]$ est une matrice réelle $(N \times N)$ diagonale déf nie positive telle que

$$
\left[\mathcal{C}_{1}\right]_{\alpha \beta}=\frac{c_{0}^{2}}{\rho_{0}}[\mathcal{M}]_{\alpha \beta}=\frac{c_{0}^{2}}{\rho_{0}} \mu_{\alpha} \delta_{\alpha \beta} .
$$

Enf n les forces généralisées s'écrivent $\mathcal{F}_{\mathrm{S}}(\omega)=[U]^{T} \mathbf{F}_{S}(\omega)$ et $\mathcal{F}_{\mathrm{Q}}(\omega)=[\Phi]^{T} \mathbf{F}_{\mathrm{Q}}(\omega)$.

\subsection{Calcul de la fonction de réponse en fréquence}

Pour calculer la fonction de réponse en fréquence (FRF) dans la bande BF, on élimine $\mathbf{r}$ dans l'Eq. [63] et l'on obtient

$$
[\widetilde{\mathcal{A}}(\omega)]\left[\begin{array}{c}
\mathbf{q}^{S} \\
\mathbf{q}
\end{array}\right]=\left[\begin{array}{c}
\mathcal{F}_{\mathrm{S}}(\omega) \\
\omega^{2} \mathcal{F}_{\mathrm{Q}}(\omega)
\end{array}\right]
$$

où la matrice complexe $[\widetilde{\mathcal{A}}(\omega)]$ est symétrique et est déf nie par

$$
\left[\begin{array}{cc}
{\left[\mathcal{A}^{S}(\omega)\right]-\omega^{2}\left[\mathcal{A}^{E}\left(\omega / c_{E}\right)\right]+\kappa(\omega)[\mathcal{J}]} & -\omega^{2}[\mathcal{C}(\omega)] \\
-\omega^{2}[\mathcal{C}(\omega)]^{T} & i \omega^{3}\left[\mathcal{D}_{\tau}(\omega)\right]+\omega^{2}[\mathcal{K}]+\omega^{2}\left[\mathcal{S}_{Z}(\omega)\right]-\omega^{4}[\mathcal{M}]
\end{array}\right] .
$$

Pour tout $\omega \neq 0$, la matrice $[\widetilde{\mathcal{A}}(\omega)]$ est inversible et l'on obtient

$$
\left[\begin{array}{c}
\mathbf{q}^{S} \\
\mathbf{q}
\end{array}\right]=[\mathcal{T}(\omega)]\left[\begin{array}{c}
\mathcal{F}_{\mathrm{S}}(\omega) \\
\omega^{2} \mathcal{F}_{\mathrm{Q}}(\omega)
\end{array}\right]
$$

où $[\mathcal{T}(\omega)]$ est une matrice complexe, $\left(\left(N_{S}+N\right) \times\left(N_{S}+N\right)\right)$, symétrique, telle que $[\mathcal{T}(\omega)]=[\widetilde{\mathcal{A}}(\omega)]^{-1}$. Le champ de déplacement dans la structure et la pression dans la cavité acoustique sont calculés en utilisant les Eqs. [62] et [56].

\subsection{Calcul des modes élastoacoustiques}

Les modes élastoacoustiques sont ceux du système conservatif associé constitué de la structure couplée avec le f uide acoustique interne, en remplacant $\kappa(\omega)$ et $[\mathcal{C}(\omega)]$ par $\kappa(0)$ et $[\mathcal{C}(0)]$ respectivement. Les Eqs. [63] et [64] donnent alors le modèle réduit matriciel symétrique

$$
\begin{gathered}
{\left[\begin{array}{ccc}
{\left[\mathcal{K}^{S}\right]+\kappa(0)[\mathcal{J}]} & {[0]} & {[0]} \\
{[0]} & {[0]} & {[0]} \\
{[0]} & {[0]} & \frac{1}{\rho_{0}^{2}}[\mathcal{M}]
\end{array}\right]\left[\begin{array}{c}
\mathbf{q}^{S} \\
\mathbf{q} \\
\mathbf{r}
\end{array}\right]} \\
=\omega^{2}\left[\begin{array}{ccc}
{\left[\mathcal{M}^{S}\right]} & {[\mathcal{C}(0)]} & {[0]} \\
{[\mathcal{C}(0)]^{T}} & -[\mathcal{K}] & \frac{1}{c_{0}^{2}}\left[\mathcal{C}_{1}\right] \\
{[0]} & \frac{1}{c_{0}^{2}}\left[\mathcal{C}_{1}\right]^{T} & {[0]}
\end{array}\right]\left[\begin{array}{c}
\mathbf{q}^{S} \\
\mathbf{q} \\
\mathbf{r}
\end{array}\right],
\end{gathered}
$$


qui correspond à un problème symétrique aux valeurs propres. En éliminant q dans l'Eq. [75], on obtient un problème symétrique généralisé aux valeurs propres dont la matrice de "masse" est déf nie positive,

$$
\left[\begin{array}{cc}
{\left[\mathcal{K}_{1}\right]} & {[0]} \\
{[0]} & {\left[\mathcal{K}_{2}\right]}
\end{array}\right]\left[\begin{array}{c}
\mathbf{q}^{S} \\
\mathbf{r}
\end{array}\right]=\omega^{2}\left[\begin{array}{cc}
{\left[\mathcal{M}_{1}\right]} & {\left[\mathcal{M}_{c}\right]} \\
{\left[\mathcal{M}_{c}\right]^{T}} & {\left[\mathcal{M}_{2}\right]}
\end{array}\right]\left[\begin{array}{c}
\mathbf{q}^{S} \\
\mathbf{r}
\end{array}\right],
$$

avec

$$
\begin{gathered}
{\left[\mathcal{K}_{1}\right]_{\alpha \beta}=\mu_{\alpha}^{S} \lambda_{\alpha}^{S} \delta_{\alpha \beta}+\kappa(0)[\mathcal{J}]_{\alpha \beta}} \\
{\left[\mathcal{K}_{2}\right]_{\alpha \beta}=\frac{\mu_{\alpha}}{\rho_{0}^{2}} \delta_{\alpha \beta} \quad, \quad\left[\mathcal{M}_{2}\right]_{\alpha \beta}=\frac{1}{\rho_{0}^{2}} \frac{\mu_{\alpha}}{\lambda_{\alpha}} \delta_{\alpha \beta}} \\
{\left[\mathcal{M}_{1}\right]_{\alpha \beta}=\mu_{\alpha}^{S} \delta_{\alpha \beta}+\sum_{\gamma=1}^{N} \frac{1}{\mu_{\gamma} \lambda_{\gamma}}[\mathcal{C}(0)]_{\alpha \gamma}[\mathcal{C}(0)]_{\beta \gamma}} \\
{\left[\mathcal{M}_{c}\right]_{\alpha \beta}=\frac{1}{\rho_{0} \lambda_{\alpha}}[\mathcal{C}(0)]_{\alpha \beta}}
\end{gathered}
$$

Les modes élastoacoustiques exprimés en termes du champ de déplacement de la structure et de la pression dans le fuide interne sont calculés en utilisant les Eqs. [62] et [56] avec $\mathbf{q}=[\mathcal{K}]^{-1}\left([\mathcal{C}(0)]^{T} \mathbf{q}^{S}+\frac{1}{c_{0}^{2}}\left[\mathcal{C}_{1}\right] \mathbf{r}\right)$ où $[\mathcal{K}]$ et $\left[\mathcal{C}_{1}\right]$ sont les matrices diagonales déf nies par les Eqs. [69] et [71]. Il est à noter que les modes élastoacoustiques (voir aussi le chap. 13 de [OHA 98]) tiennent compte des effets de raideur de l'impédance de paroi sur $\Gamma_{Z}$ (effets de raideur non pris en compte dans le chap. 9 de [MOR 95]).

Ces modes élastoacoustiques peuvent être utilisés pour calculer la fonction de réponse en fréquence en incluant les termes de correction quasi-statique pour accélérer la convergence de la projection modale. Il est à noter que le problème aux limites décrit au paragraphe 5.2 est bien posé pour le problème statique (fréquence nulle). Toutefois, la méthode présentée au paragraphe 5.5 est préférable, que le f uide acoustique interne soit un gaz ou un liquide.

\section{Modélisation, discrétisation par éléments finis, méthode de réduction en vibroacoustique moyennes fréquences}

On considère le problème décrit au paragraphe 3 (voir Figure 2). Contrairement au domaine BF, le calcul des modes élastoacoustiques et la construction de modèles réduits modaux ne sont pas praticables pour le domaine MF. Dans ces conditions, on peut choisir pour le couplage de la structure avec le f uide interne une formulation ne faisant intervenir qu'un seul champ scalaire inconnu pour le f uide tout en préservant la symétrie des matrices globales pour le problème couplé. Il a été démontré que pour le problème considéré, le champ scalaire pour le f uide interne dissipatif est un champ relié au potentiel des vitesses. Concernant les détails des développements qui suivent, on renvoie le lecteur au chap. 14 de [OHA 98].

\subsection{Expression du champ de pression dans le fluide acoustique interne}

On introduit alors le champ scalaire $\psi$ relié à $\varphi$ introduit au paragraphe $5.1 \operatorname{par} \psi=i \omega \varphi$. Le potentiel des vitesses $\widetilde{\psi}$ s'écrit $\widetilde{\psi}=i \omega \widetilde{\varphi}$, c'est-à-dire en utilisant l'Eq. [22], $\widetilde{\psi}(\mathbf{x}, \omega)=(1+i \omega \tau) \psi(\mathbf{x}, \omega)+\left(\tau c_{0}^{2} / \rho_{0}\right) \frac{Q(\mathbf{x}, \omega)}{i \omega}$. Dans ces conditions (voir [OHA 98]) on a

$$
p(\mathbf{x}, \omega)=-i \omega \rho_{o} \psi(\mathbf{x}, \omega)+\pi(\omega ; \mathbf{u} ; \psi) \quad,
$$

où le champ $\psi$ doit être tel que

$$
\int_{\Omega} \psi d \mathbf{x}=0
$$

et où $\pi(\omega ; \mathbf{u} ; \psi)$ s'écrit

$$
\pi(\omega ; \mathbf{u} ; \psi)=\kappa(\omega)\left\{i \omega \rho_{0} \pi_{1}(\omega ; \psi)-\pi_{2}(\mathbf{u})+\pi_{\mathrm{Q}}(\omega)\right\} \quad .
$$


Dans l'Eq. [81], $\pi_{1}(\omega ; \psi), \pi_{2}(\mathbf{u})$ et $\pi_{\mathrm{Q}}(\omega)$ sont donnés par

$$
\begin{gathered}
\pi_{1}(\omega ; \psi)=\int_{\Gamma_{Z}} \frac{\psi(\mathbf{x}, \omega)}{i \omega Z(\mathbf{x}, \omega)} d s(\mathbf{x}), \\
\pi_{2}(\mathbf{u})=\int_{\Gamma \cup \Gamma_{Z}} \mathbf{u}(\mathbf{x}, \omega) \cdot \mathbf{n}(\mathbf{x}) d s(\mathbf{x}), \\
\pi_{\mathrm{Q}}(\omega)=\frac{1}{\rho_{0}} \int_{\Omega} \frac{Q(\mathbf{x}, \omega)}{i \omega} d \mathbf{x} .
\end{gathered}
$$

Dans l'Eq. [81], $\kappa(\omega)$ est un nombre complexe donné par

$$
\kappa(\omega)=\frac{\rho_{0} c_{0}^{2}}{|\Omega|} \times\left\{1+\frac{\rho_{0} c_{0}^{2}}{|\Omega|} \int_{\Gamma_{Z}} \frac{d s(\mathbf{x})}{i \omega Z(\mathbf{x}, \omega)}\right\}^{-1}
$$

où $|\Omega|$ désigne le volume du domaine $\Omega$.

\subsection{Problème aux limites pour le système vibroacoustique}

Equations pour la structure. L'équation d'équilibre dynamique pour la structure $\Omega_{S}$ s'écrit

$$
-\omega^{2} \rho_{S} u_{i}-\sigma_{i j, j}(\mathbf{u})=g_{i} \quad \text { dans } \quad \Omega_{S},
$$

où $\rho_{S}(\mathbf{x})$ est la masse volumique de la structure. Dans l'Eq. [86], la loi de comportement pour le domaine MF s'écrit (voir le chap. 4 de [OHA 98])

$$
\sigma_{i j}(\mathbf{x}, \omega)=\left(a_{i j k h}(\mathbf{x}, \omega)+i \omega b_{i j k h}(\mathbf{x}, \omega)\right) \varepsilon_{k h}(\mathbf{x}, \omega)
$$

où $\varepsilon_{k h}(\mathbf{x}, \omega)=\frac{1}{2}\left(u_{k, h}+u_{h, k}\right)$ est le tenseur de déformation linéarisé. La condition aux limites sur $\Gamma_{E} \mathbf{s}$ 'écrit $\sigma_{i j}(\mathbf{u}) n_{j}^{S}=G_{i}-\left.p_{E}\right|_{\Gamma_{E}} n_{i}^{S}$, soit en utilisant l'Eq. [18],

$$
\sigma_{i j}(\mathbf{u}) n_{j}^{S}=G_{i}-\left.p_{\text {donnée }}\right|_{\Gamma_{E}} n_{i}^{S}-i \omega \mathbf{Z}_{\Gamma_{E}}(\omega)\left\{\mathbf{u} \cdot \mathbf{n}^{S}\right\} n_{i}^{S} \quad \text { sur } \quad \Gamma_{E} .
$$

La condition aux limites sur $\Gamma \cup \Gamma_{Z}$ s'écrit $\sigma_{i j}(\mathbf{u}) n_{j}^{S}=G_{i}-p(\omega) n_{i}^{S}=G_{i}+p(\omega) n_{i}$ où $\mathbf{n}=-\mathbf{n}^{S}$, soit en utilisant l'Eq. [79],

$$
\sigma_{i j}(\mathbf{u}) n_{j}^{S}=G_{i}+\kappa \pi_{\mathrm{Q}} n_{i}-i \omega \rho_{0} \psi n_{i}+i \omega \rho_{0} \kappa \pi_{1}(\omega ; \psi) n_{i}-\kappa \pi_{2}(\mathbf{u}) n_{i} \quad \text { sur } \quad \Gamma \cup \Gamma_{Z} .
$$

Equations pour le fluide acoustique interne dissipatif. Le champ $\psi(\mathbf{x}, \omega)$, qui vérif e la contrainte déf nie par l'Eq. [80], satisfait l'équation suivante

$$
\begin{gathered}
-\omega^{2} \frac{\rho_{0}}{c_{0}^{2}} \psi(\mathbf{x}, \omega)-i \omega \tau \rho_{0} \nabla^{2} \psi(\mathbf{x}, \omega)-\rho_{0} \nabla^{2} \psi(\mathbf{x}, \omega)= \\
i \omega g_{\mathrm{Q}}-\frac{\omega^{2} \rho_{0} \kappa}{c_{0}^{2}} \pi_{1}(\omega ; \psi)-\frac{i \omega \kappa}{c_{0}^{2}} \pi_{2}(\mathbf{u}) \text { dans } \Omega,
\end{gathered}
$$

où $g_{\mathrm{Q}}$ est déf ni par l'Eq. [93] ci-dessous. La condition de Neumann sur $\Gamma$ s'écrit

$$
\rho_{0}(1+i \omega \tau) \frac{\partial \psi}{\partial \mathbf{n}}=i \omega \rho_{0} \mathbf{u} \cdot \mathbf{n}+i \omega G_{\mathrm{Q}} \quad \text { sur } \quad \Gamma
$$

avec $G_{Q}$ déf ni par l'Eq. [94] ci-dessous. La frontière $\Gamma_{Z}$ (voir Figure 2) a des propriétés acoustiques modélisées par une impédance acoustique de paroi $Z(\mathbf{x}, \omega)$ à valeurs complexes qui est déf nie, comme en BF, par l'Eq. [42]. La condition de Neumann sur $\Gamma_{Z}$ en présence de l'impédance acoustique de paroi s'écrit

$$
\begin{aligned}
& \rho_{0}(1+i \omega \tau) \frac{\partial \psi}{\partial \mathbf{n}}=i \omega \rho_{0} \mathbf{u} \cdot \mathbf{n}+\frac{\omega^{2} \rho_{0}^{2}}{i \omega Z} \psi \\
& \quad-\frac{\omega^{2} \rho_{0}^{2} \kappa}{i \omega Z} \pi_{1}(\omega ; \psi)-\frac{i \omega \kappa \rho_{0}}{i \omega Z} \pi_{2}(\mathbf{u})+i \omega G_{\mathrm{Q}}+i \omega G_{\mathrm{Q}, \mathrm{Z}} \quad \operatorname{sur} \Gamma_{Z},
\end{aligned}
$$


avec $G_{\mathrm{Q}, \mathrm{Z}}$ déf ni par l'Eq. [95] ci-dessous. La fonction $g_{\mathrm{Q}}$ est déf nie dans $\Omega$ par

$$
g_{\mathrm{Q}}(\mathbf{x}, \omega)=\frac{1}{c_{0}^{2}} \kappa(\omega) \pi_{\mathrm{Q}}(\omega)-\frac{1}{i \omega} Q(\mathbf{x}, \omega)-\tau c_{0}^{2} \frac{1}{\omega^{2}} \nabla^{2} Q(\mathbf{x}, \omega)
$$

$G_{\mathrm{Q}}$ est déf nie sur $\Gamma \cup \Gamma_{Z}$ par

$$
G_{\mathrm{Q}}(\mathbf{x}, \omega)=\tau c_{0}^{2} \frac{1}{\omega^{2}} \frac{\partial Q(\mathbf{x}, \omega)}{\partial \mathbf{n}(\mathbf{x})}
$$

et $G_{\mathrm{Q}, \mathrm{Z}}$ est déf nie sur $\Gamma_{Z}$ par

$$
G_{\mathrm{Q}, \mathrm{Z}}(\mathbf{x}, \omega)=\frac{\rho_{0}}{i \omega Z(\mathbf{x}, \omega)} \kappa(\omega) \pi_{\mathrm{Q}}(\omega)
$$

\subsection{Discrétisation par éléments finis}

On considère un maillage éléments f nis de la structure $\Omega_{S}$ et du f uide interne $\Omega$, les deux maillages étant supposés compatibles sur l'interface $\Gamma \cup \Gamma_{Z}$. Soit $\mathbf{U}=\left(U_{1}, \ldots, U_{n_{S}}\right)$ le vecteur complexe des $n_{S}$ DDL de la structure qui sont les valeurs nodales du champ de déplacement $\mathbf{u}$. Soit $\Psi=\left(\Psi_{1}, \ldots, \Psi_{n}\right)$ le vecteur complexe des $n$ DDL du f uide qui sont les valeurs nodales du champ $\psi$. La discrétisation par éléments f nis de la formulation variationnelle en $\mathbf{u}$ et $\psi$ du problème aux limites déf ni par les Eqs. [86] à [95], conduit au système complexe symétrique suivant

$$
[A(\omega)]\left[\begin{array}{l}
\mathbf{U} \\
\mathbf{\Psi}
\end{array}\right]=\left[\begin{array}{c}
\mathbf{F}_{\mathrm{S}}(\omega) \\
-i \omega \mathbf{F}_{\mathrm{Q}}(\omega)
\end{array}\right]
$$

avec la contrainte correspondant à la discrétisation par éléments f nis de l’Eq. [80]

$$
\mathbf{L}^{T} \boldsymbol{\Psi}=0
$$

où $\mathbf{L}$ est un vecteur de $\mathbb{R}^{n}$. La matrice complexe symétrique $[\mathbb{A}(\omega)]$ est déf nie par

$$
[\mathbb{A}(\omega)]=\left[\begin{array}{cc}
{\left[A^{S}(\omega)\right]-\omega^{2}\left[A^{E}\left(\omega / c_{E}\right)\right]+\kappa(\omega)[J]} & i \omega[C(\omega)] \\
i \omega[C(\omega)]^{T} & -\left[A^{F}(\omega)\right]
\end{array}\right],
$$

où $\left[A^{S}(\omega)\right]$ est la matrice complexe symétrique $\left(n_{S} \times n_{S}\right)$ de raideur dynamique de la structure (qui n'est singulière que pour $\omega=0$ de par la présence des modes de corps rigide) qui s'écrit

$$
\left[A^{S}(\omega)\right]=-\omega^{2}\left[M^{S}\right]+i \omega\left[D^{S}(\omega)\right]+\left[K^{S}(\omega)\right]
$$

où $\left[M^{S}\right],\left[D^{S}(\omega)\right]$ et $\left[K^{S}(\omega)\right]$ les matrices $\left(n_{S} \times n_{S}\right)$ réelles symétriques de masse, de dissipation et de raideur de la structure in vacuo, la matrice $\left[M^{S}\right]$ étant déf nie positive et les matrices $\left[D^{S}(\omega)\right]$ et $\left[K^{S}(\omega)\right]$ étant positives (et ayant le même noyau). On notera que, contrairement à la modélisation $\mathrm{BF}$, la matrice de raideur dépend de $\omega$ en MF. La matrice $\left(n_{S} \times n_{S}\right)$ complexe symétrique $\left[A^{E}\left(\omega / c_{E}\right)\right]$ qui traduit les effets du f uide extérieur sur la structure, est celle déf nie par l'Eq. [51] et la matrice $\left(n_{S} \times n_{S}\right)$ réelle symétrique [ $J$ ] est celle déf nie par l'Eq. [52]. La matrice $(n \times n)$ complexe symétrique $\left[A^{F}(\omega)\right]$ est inversible pour tout $\omega \neq 0$ et s'écrit

$$
\left[A^{F}(\omega)\right]=-\omega^{2}[M]+i \omega\left[D_{\tau}(\omega)\right]+[K]+\left[S_{Z}(\omega)\right] .
$$

Les matrices $(n \times n)$ réelles $[M],[K]$ et $\left[D_{\tau}(\omega)\right]$ et la matrice $(n \times n)$ complexe $\left[S_{Z}(\omega)\right]$ sont les matrices déf nies au paragraphe 5.3. La matrice rectangulaire $\left(n_{S} \times n\right)$ complexe $[C(\omega)]$ est déf nie par l'Eq. [54]. Le lecteur trouvera les expressions (en fonction des données) du second membre de l'Eq. [96] dans les chap. 11 et 14 de [OHA 98]. Soit $\mathbf{P}=\left(p_{1}, \ldots, p_{n}\right)$ le vecteur des DDL constitués des valeurs de la pression $p$ aux $n_{p}$ noeuds du maillage du f uide interne $\Omega$. Il est calculé par

$$
\mathbf{P}=-i \omega \rho_{0} \boldsymbol{\Psi}+\kappa(\omega)\left\{i \omega \rho_{0} \boldsymbol{\Pi}_{1}(\omega)^{T} \boldsymbol{\Psi}-\boldsymbol{\Pi}_{2}^{T} \mathbf{U}+\pi_{\mathrm{Q}}(\omega)\right\} \frac{\rho_{0}}{c_{0}^{2}}[M]^{-1} \mathbf{L}
$$




\subsection{Introduction d'une méthode à deux échelles de temps, dite méthode MF, pour le calcul des fonctions de réponse en fréquence dans le domaine des moyennes fréquences}

Conformément à ce qui a été indiqué dans le paragraphe 2 , nous présentons ci-après une méthode à deux échelles de temps [SOI 82] (dite méthode MF) qui permet de résoudre l'Eq. [96] sans avoir à résoudre le système linéaire fréquence par fréquence, ce qui n'est pas praticable. Nous en présentons les grandes lignes et renvoyons le lecteur aux chap. 7 et 14 de [OHA 98] pour les détails. Soit $\mathbb{B}_{\mathrm{MF}}$ la bande large MF d'analyse déf nie par

$$
\mathbb{B}_{\mathrm{MF}}=\left[\omega_{\mathrm{MF}, \text { init }}, \omega_{\mathrm{MF}, \text { final }}\right] \text {. }
$$

On considère une bande étroite MF notée $\mathbb{B}_{\nu}$ et déf nie par

$$
\mathbb{B}_{\nu}=\left[\Omega_{\nu}-\Delta \omega / 2, \Omega_{\nu}+\Delta \omega / 2\right],
$$

où $\Omega_{\nu}>0$ est la fréquence centrale de la bande $\mathbb{B}_{\nu}$ et $\Delta \omega$ sa largeur de bande telle que $0<\Delta \omega<2 \Omega_{\nu}$. On associe à la bande $\mathbb{B}_{\nu}$ la bande de même largeur $\mathbb{B}_{0}$ telle que

$$
\mathbb{B}_{0}=[-\Delta \omega / 2, \Delta \omega / 2] .
$$

Par déf nition la bande $\mathbb{B}_{\nu}$ est une bande étroite MF si $\Delta \omega / \Omega_{\nu} \ll 1$. On introduit alors deux échelles de temps $\tau_{\text {long }}$ et $\tau_{\text {court }}$ telles que $\tau_{\text {long }}=2 \pi / \Delta \omega$ et $\tau_{\text {court }}=2 \pi / \Omega_{\nu}$. On a $\tau_{\text {court }} / \tau_{\text {long }}=\Delta \omega / \Omega_{\nu} \ll 1$. En conséquence, $\tau_{\text {court }}$ représente une échelle de temps courte associée à la fréquence centrale $\Omega_{\nu}$ de la bande MF et $\tau_{\text {long }}$ représente une échelle de temps longue associée à la largeur $\Delta \omega$ de la bande MF étroite $\mathbb{B}_{\nu}$. La bande $\mathbb{B}_{0}$ est appelée la bande $\mathrm{BF}$ associée à la bande $\mathrm{MF}$ étroite $\mathbb{B}_{\nu}$ car elle ne fait intervenir que l'échelle de temps longue $\tau_{\text {long }}$. Pour tout $\omega$ dans $\mathbb{B}_{\mathrm{MF}}$, on a à construire la FRF $[\mathbb{T}(\omega)]=[\mathbb{A}(\omega)]^{-1}$ où $[\mathbb{A}(\omega)]$ est déf nie par l'Eq. [98]. Pour construire cette FRF, on réecrit les Eqs. [96] et [97] comme suit

$$
[\mathbb{A}(\omega)] \mathbf{Y}(\omega)=\theta_{\nu}(\omega) \mathbf{B} \quad, \quad \omega \in \mathbb{B}_{\nu} \quad \text { avec } \quad \mathbf{Y}(\omega)=\left[\begin{array}{l}
\mathbf{U}(\omega) \\
\mathbf{\Psi}(\omega)
\end{array}\right],
$$

avec la contrainte

$$
\mathbf{L}^{T} \mathbf{\Psi}(\omega)=0 \quad, \quad \omega \in \mathbb{B}_{\nu},
$$

le second membre de l'Eq. [105] correspondant à une classe particulière d'excitation relative à la bande MF étroite $\mathbb{B}_{\nu}$. Le vecteur complexe $\mathbf{B}$ est indépendant de $\omega$ et $\theta_{\nu}(\omega)$ est une fonction à valeur complexe de carré intégrable sur $\mathbb{R}$ telle que

$$
\theta_{\nu}(\omega)=0 \quad, \quad \forall \omega \notin \mathbb{B}_{\nu} .
$$

Soit $\theta_{\nu}(t)$ la fonction de $\mathbb{R}$ dans $\mathbb{C}$ déf nie comme la transformée de Fourier inverse $\theta_{\nu}(t)=$ $(2 \pi)^{-1} \int_{\mathbb{B}_{\nu}} e^{i \omega t} \theta_{\nu}(\omega) d \omega$. On a donc $\theta_{\nu}(\omega)=\int_{\mathbb{R}} e^{-i \omega t} \theta_{\nu}(t) d t$. Le signal BF $\theta_{0}(t)$ associé à $\theta_{\nu}(t)$ est déf ni par

$$
\theta_{0}(t)=\theta_{\nu}(t) e^{-i \Omega_{\nu} t} .
$$

En prenant la transformée de Fourier de l’Eq. [108], on en déduit que

$$
\theta_{0}(\omega)=\theta_{\nu}\left(\omega+\Omega_{\nu}\right) \quad, \quad \forall \omega \in \mathbb{R} .
$$

Par conséquent, $\theta_{0}(\omega)=0$ pour tout $\omega \notin \mathbb{B}_{0}$ et donc $\theta_{0}(t)$ est un signal $\mathrm{BF}$ sur la bande $\mathbb{B}_{0}$ qui n'a que l'échelle de temps longue $\tau_{\text {long }}$. La matrice symétrique complexe $[A(\omega)]$ déf nie par l'Eq. [98] peut s'écrire

$$
[\mathbb{A}(\omega)]=-\omega^{2}[\mathbb{M}(\omega)]+i \omega[\mathbb{D}(\omega)]+[\mathbb{K}(\omega)],
$$

où $[\mathbb{M}(\omega)],[\mathbb{D}(\omega)]$ et $[\mathbb{K}(\omega)]$ sont des matrices symétriques complexes déf nies par

$$
[M(\omega)]=\left[\begin{array}{cc}
{\left[M^{S}\right]+\left[A^{E}\left(\omega / c_{E}\right)\right]} & {[0]} \\
{[0]} & -[M]
\end{array}\right],
$$




$$
\begin{aligned}
& {[\mathbb{D}(\omega)]=\left[\begin{array}{lc}
{\left[D^{S}(\omega)\right]} & {[C(\omega)]} \\
{[C(\omega)]^{T}} & -\left[D_{\tau}(\omega)\right]-\left[S_{z}^{I}(\omega)\right]
\end{array}\right],} \\
& {[\mathbb{K}(\omega)]=\left[\begin{array}{cc}
{\left[K^{S}(\omega)\right]+\kappa(\omega)[J]} & {[0]} \\
{[0]} & -[K]-\left[S_{z}^{R}(\omega)\right]
\end{array}\right],}
\end{aligned}
$$

avec

$$
\left[S_{z}(\omega)\right]=\left[S_{z}^{R}(\omega)\right]+i \omega\left[S_{z}^{I}(\omega)\right]
$$

où les matrices $\left[S_{z}^{R}(\omega)\right]$ et $\left[S_{z}^{I}(\omega)\right]$ sont telles que $\left[S_{z}^{R}(\omega)\right]=\Re e\left[S_{z}(\omega)\right]$ et $\omega\left[S_{z}^{I}(\omega)\right]=\Im m\left[S_{z}(\omega)\right]$. Pour construire la FRF, on prend $\theta_{\nu}(\omega)=1$ pour $\omega \in \mathbb{B}_{\nu}$ et $\mathbf{B}$ représente les vecteurs de la base canonique de $\mathbb{R}^{n_{S}+n}$. La méthode proposée permet de calculer la réponse pour différents types d'excitations déterministes et aléatoires, dès que la solution du problème déf ni par les Eqs. [105] et [106] est construite; nous renvoyons le lecteur à [OHA 98] pour les détails. Dans la méthode MF, on remplace l'Eq. [105] par l'approximation

$$
\left(-\omega^{2}\left[\mathbb{M}_{\nu}\right]+i \omega\left[\mathbb{D}_{\nu}\right]+\left[\mathbb{K}_{\nu}\right]\right) \mathbf{Y}_{\nu}(\omega)=\theta_{\nu}(\omega) \mathbf{B} \quad, \quad \omega \in \mathbb{B}_{\nu},
$$

où les matrices complexes $\left[\mathbb{M}_{\nu}\right],\left[\mathbb{D}_{\nu}\right]$ et $\left[\mathbb{K}_{\nu}\right]$ dépendent de la bande $\mathbb{B}_{\nu}$ mais sont constantes à l'intérieur de la bande et sont telles que

$$
\left[\mathbb{M}_{\nu}\right]=\left[\mathbb{M}\left(\Omega_{\nu}\right)\right] \quad, \quad\left[\mathbb{D}_{\nu}\right]=\left[\mathbb{D}\left(\Omega_{\nu}\right)\right] \quad, \quad\left[\mathbb{K}_{\nu}\right]=\left[\mathbb{K}\left(\Omega_{\nu}\right)\right] .
$$

On montre, moyennant des hypothèses usuellement vérif ées par les applications, que $\omega \mapsto[\mathbb{M}(\omega)]$, $[\mathbb{D}(\omega)]$ et $[\mathbb{K}(\omega)]$ sont des fonctions lentement variables sur la bande MF étroite $\mathbb{B}_{\nu}$ (voir [OHA 98]). On introduit le problème de Cauchy temporel

$$
\left[\mathbb{M}_{\nu}\right] \ddot{\mathbf{Y}}_{0}(t)+\left[\widetilde{\mathbb{D}}_{\nu}\right] \dot{\mathbf{Y}}_{0}(t)+\left[\widetilde{\mathbb{K}}_{\nu}\right] \mathbf{Y}_{0}(t)=\theta_{0}(t) \mathbf{B} \text { pour } t_{\mathrm{i}}<t \leq t_{\mathrm{f}},
$$

avec la contrainte

$$
\mathbf{L}^{T} \boldsymbol{\Psi}_{0}(t)=0 \quad, \quad \forall t>t_{\mathrm{i}} \quad,
$$

et les conditions initiales

$$
\mathbf{Y}_{0}\left(t_{\mathrm{i}}\right)=\mathbf{0} \quad, \quad \dot{\mathbf{Y}}_{0}\left(t_{\mathrm{i}}\right)=\mathbf{0}
$$

où $\mathbf{Y}_{0}(t)$ est tel que

$$
\mathbf{Y}_{0}(t)=\left[\begin{array}{l}
\mathbf{U}_{0}(t) \\
\mathbf{\Psi}_{0}(t)
\end{array}\right],
$$

et où les matrices complexes symétriques $\left[\widetilde{\mathbb{D}}_{\nu}\right]$ et $\left[\widetilde{\mathbb{K}}_{\nu}\right]$ sont telles que

$$
\left[\widetilde{\mathbb{D}}_{\nu}\right]=\left[\mathbb{D}_{\nu}\right]+2 i \Omega_{\nu}\left[\mathbb{M}_{\nu}\right] \quad, \quad\left[\widetilde{\mathbb{K}}_{\nu}\right]=-\Omega_{\nu}^{2}\left[\mathbb{M}_{\nu}\right]+i \Omega_{\nu}\left[\mathbb{D}_{\nu}\right]+\left[\mathbb{K}_{\nu}\right] .
$$

On montre alors que la solution du problème MF déf ni par les Eqs. [105] et [106] peut être approchée d'aussi près que l'on veut par

$$
\mathbf{Y}_{\nu}(\omega) \simeq \mathbf{1}_{\mathbb{B}_{\nu}}(\omega) \Delta t \sum_{m=m_{\mathrm{i}}}^{m_{\mathrm{f}}} \mathbf{Y}_{0}(m \Delta t) e^{-i m \Delta t\left(\omega-\Omega_{\nu}\right)} \quad, \quad \forall \omega \in \mathbb{R}
$$

avec $\mathbf{1}_{\mathbb{B}_{\nu}}(\omega)=1$ si $\omega \in \mathbb{B}_{\nu}$ et $=0$ si $\omega \notin \mathbb{B}_{\nu}$ et où les entiers $m_{\mathrm{i}}$ et $m_{\mathrm{f}}$ sont tels que $t_{\mathrm{i}}=m_{\mathrm{i}} \Delta t$ et $t_{\mathrm{f}}=m_{\mathrm{f}} \Delta t$ avec $\Delta t=2 \pi / \Delta \omega$. L'entier négatif $m_{\mathrm{i}}$ et l'entier positif $m_{\mathrm{f}}$ sont déterminés avec des critères précis que l'on trouvera dans le chap. 7 de [OHA 98]. Donnons quelques indications sur le choix de ces paramètres. L'expérience acquise montre que $m_{\mathrm{i}}=-3$ est un bon compromis pour le choix correspondant à $\theta_{0}(\omega)=\mathbf{1}_{\mathbb{E}_{0}}(\omega)$, c'est-à dire $\theta_{0}(t)=(\pi t)^{-1} \sin (t \Delta \omega / 2)$. En ce qui concerne $m_{\mathrm{f}}$, on montre que sa valeur peut être estimée par la formule

$$
m_{\mathrm{f}}=\frac{\ln 10}{2 \pi \xi_{\nu}\left(\frac{1}{\Delta \omega / \Omega_{\nu}}-\frac{1}{2}\right)},
$$


où $\xi_{\nu}$ désigne le taux de dissipation moyen du système mécanique sur la bande $\mathbb{B}_{\nu}$. Typiquement pour $\Delta \omega / \Omega_{\nu}=1 / 10$ et $\xi_{\nu} \simeq 0.01$, on a $m_{\mathrm{f}} \simeq 4$. Il est à noter que la solution MF est calculée directement par l'Eq. [122] à partir de la seule connaissance de la solution $\mathbf{Y}_{0}(t)$ du problème BF déf ni par les Eqs. [117] à (119), problème qui est résolu classiquement à l'aide d'un algorithme d'intégration temporel pas à pas implicite inconditionnellement stable du type Newmark. En ce qui concerne le pas de temps $\delta t$ d'intégration d'un tel schéma, il doit être tel que $\delta t=\Delta t / p$ avec $p>1$. L'expérience montre que $p=3$ est une valeur appropriée. La très grande eff cacité de cette méthode provient du fait que, d'une part, l'échelle de temps courte est traitée exactement (analytiquement) à coût numérique nul et que, d'autre part, seule l'échelle de temps longue est traitée numériquement par des algorithmes classiques (résolution du problème BF associé). De plus, pour les bandes MF et même en présence de dissipation faible, plus $\Delta \omega / \Omega_{\nu}$ sera petit et plus le nombre de pas de temps $\delta t$ sera faible. Typiquement, on voit donc que, pour $\Delta \omega / \Omega_{\nu}=1 / 10$ et $\xi_{\nu} \simeq 0.01$, toute la dynamique en module et en phase est obtenue pour tout $\omega$ dans $\mathbb{B}_{\nu}$ avec seulement $7 \times p$ pas de temps, c'est-à-dire de l'ordre de 20 pas de temps pour $p=3$. Enf n signalons que la structure algébrique particulière des matrices $[J]$ et $\left[S_{Z}(\omega)\right]$ permet de décomposer la matrice $[A(\omega)]$ déf nie par l'Eq. [110] comme la somme d'une matrice creuse avec une matrice de la forme $\Gamma \Gamma^{T}$ où $\Gamma$ est un vecteur. On peut alors mettre en oeuvre une procédure numérique spécif que pour diminuer les coûts numériques (voir chap. 14 de [OHA 98]).

\subsection{Modèle réduit matriciel symétrique}

Dans le contexte de la construction d'un modèle réduit en dynamique MF, la problématique est la suivante. Comment peut-on remplacer pour le calcul de la réponse dans la bande MF, la base modale de la structure (méthode de type BF qui devient impraticable en MF), par une autre base qui soit calculable et adaptée à la bande MF?

On propose [SOI 98] une méthode qui consiste à prendre la base du sous-espace propre dominant de l'opérateur d'énergie associé à la bande MF. Par mesure de simplif cation, nous limiterons l'exposé au cas d'une structure non couplée avec des f uides acoustiques af $\mathrm{n}$ de présenter simplement les concepts. Pour le cas d'une structure in vacuo, l'Eq. [96] se réduit à

$$
\left[A^{S}(\omega)\right] \mathbf{U}(\omega)=\mathbf{F}_{S}(\omega)
$$

Cette équation correspond à la discrétisation par éléments f nis de la formulation variationnelle du problème continu pour la structure dont l'équation abstraite s'écrit $\mathbf{A}^{S}(\omega) \mathbf{u}(\omega)=\mathbf{f}_{S}(\omega)$. On considère la bande MF notée $\mathbb{B}_{\nu}$ (cf. Eq. [103]) et on introduit l'opérateur d'énergie mécanique totale $\mathbf{E}_{\mathbb{B}_{\nu}}$ sur la bande $\mathbb{B}_{\nu}$ des vibrations de la structure dissipative. On montre que cet opérateur est symétrique déf ni positif, qu'il a un ensemble dénombrable de valeurs propres positives, que les fonctions propres correspondantes forment une base réelle complète de l'espace admissible $V$ des champs de déplacement de la structure, et enf n que la série des valeurs propres est sommable. Cette dernière propriété permet de construire une stratégie d'approximation en considérant le sous-espace vectoriel $V_{N}$ de $V$ engendré par les $N$ fonctions propres associées aux $N$ plus grandes valeurs propres (sous-espace propre dominant). On obtient le modèle matriciel réduit relatif à la bande MF considérée en projetant l'Eq. [124] sur cette base. Il est important de noter que, d'une part, la base réelle utilisée tient compte de la dissipation du système mécanique (propriété essentielle pour le domaine MF) et d'autre part, que la dimension du sous-espace propre dominant reste petite (quelques dizaines en pratique pour des bandes MF étroites). On donne ci-après les grandes lignes de la méthode. Pour les détails, on renvoie le lecteur au chap. 8 de [OHA 98] et à [SOI 98].

\subsubsection{Introduction de l'opérateur d'énergie et discrétisation par éléments fnis}

Supposons provisoirement que $\mathbf{f}_{S}(\omega)$ s'écrive $\mathbf{f}_{S}(\omega)=\theta_{\nu}(\omega) \mathbf{f}$ avec $\theta_{\nu}$ déf nie au paragraphe 6.4, telle que $\left|\theta_{\nu}(-\omega)\right|=\left|\theta_{\nu}(\omega)\right|$ et où $\mathbf{f}$ est indépendant de la fréquence (pour la discrétisation par éléments f nis on aura donc $\mathbf{F}_{S}(\omega)=\theta_{\nu}(\omega) \mathbf{F}$ avec $\mathbf{F}$ un vecteur complexe indépendant de la fréquence). Cette hypothèse est utilisée uniquement pour construire la base fonctionnelle de réduction adaptée au domaine MF. Une fois le modèle réduit MF obtenu avec cette base, la réponse du système mécanique pourra être calculée avec le modèle réduit pour toute excitation déterministe ou aléatoire n'utilisant pas cette hypothèse restrictive (mais nécessaire pour la construction de la base fonctionnelle af $\mathrm{n}$ d'obtenir un modèle réduit valable pour tout type d'excitation). Pour tout $\omega$ f xé dans $\mathbb{B}_{\nu} \cup \widetilde{\mathbb{B}}_{\nu}$, avec $\widetilde{\mathbb{B}}_{\nu}=\left[-\Omega_{\nu}-\Delta \omega / 2,-\Omega_{\nu}+\Delta \omega / 2\right]$, le problème variationnel a une unique 
solution $\mathbf{u}^{\mathbf{f}}(\omega)$ à valeurs dans l'espace admissible $V$ et s'écrit

$$
\mathbf{u}^{\mathbf{f}}(\omega)=\theta_{\nu}(\omega) \mathbf{T}^{S}(\omega) \mathbf{f}
$$

où $\mathbf{T}^{S}(\omega)$ est l'opérateur de réponse en fréquence dont la discrétisation par éléments f nis s'écrit

$$
\left[T^{S}(\omega)\right]=\left[A^{S}(\omega)\right]^{-1} \quad \text { avec } \quad\left[T^{S}(\omega)\right]^{*}=\overline{\left[T^{S}(\omega)\right]},
$$

où le symbole $*$ désigne la matrice adjointe et la barre désigne la conjugaison. L'énergie $\varepsilon_{\mathbb{B}_{\nu}}\left(\mathbf{u}^{\mathbf{f}}\right)$ de la vibration $\mathbf{u}^{\mathbf{f}}$ donnée par l'Eq. [125] est déf nie comme deux fois la valeur de l'énergie cinétique totale de la structure, c'est-à-dire en utilisant la formule de Plancherel,

$$
\varepsilon_{\mathbb{B}_{\nu}}\left(\mathbf{u}^{\mathbf{f}}\right)=\frac{1}{2 \pi} \int_{\mathbb{B}_{\nu} \cup \widetilde{\mathbb{B}}_{\nu}} \omega^{2} m^{S}\left(\mathbf{u}^{\mathbf{f}}(\omega), \mathbf{u}^{\mathbf{f}}(\omega)\right) d \omega,
$$

avec $m^{S}(\mathbf{u}, \mathbf{v})=\int_{\Omega_{S}} \rho_{S} \mathbf{u}(\mathbf{x}, \omega) \cdot \overline{\mathbf{v}(\mathbf{x}, \omega)} d \mathbf{x}$. Soient $\mathbf{u}^{\mathbf{f}}(\omega)=\theta_{\nu}(\omega) \mathbf{T}^{S}(\omega) \mathbf{f}$ et $\mathbf{u}^{\mathbf{g}}(\omega)=\theta_{\nu}(\omega) \mathbf{T}^{S}(\omega) \mathbf{g}$ les réponses dues aux excitations $\theta_{\nu}(\omega) \mathbf{f}$ et $\theta_{\nu}(\omega) \mathbf{g}$ respectivement, où $\mathbf{f}$ et $\mathbf{g}$ sont toujours indépendants de $\omega$. L'opérateur d'énergie $\mathbf{E}_{\mathbb{B}_{\nu}}$ relatif à la bande $\mathbb{B}_{\nu}$ est déf ni par

$$
\ll \mathbf{E}_{\mathbb{B}_{\nu}} \mathbf{f}, \mathbf{g} \gg=\frac{1}{2 \pi} \int_{\mathbb{B}_{\nu} \cup \widetilde{B}_{\nu}} \omega^{2} m^{S}\left(\mathbf{u}^{\mathbf{f}}(\omega), \mathbf{v}^{\mathbf{f}}(\omega)\right) d \omega .
$$

On déduit alors que $\varepsilon_{\mathbb{B}_{\nu}}\left(\mathbf{u}^{\mathbf{f}}\right)=\ll \mathbf{E}_{\mathbb{B}_{\nu}} \mathbf{f}, \mathbf{f} \gg$. Il est à noter que l'opérateur $\mathbf{E}_{\mathbb{B}_{\nu}}$ depend de la bande $\mathbb{B}_{\nu}$ et de $\theta_{\nu}$, mais est indépendant de $\mathbf{f}$ et $\mathbf{g}$. On montre que $\mathbf{E}_{\mathbb{B}_{\nu}}$ est réel, symétrique, déf ni positif, dont l'image est un sous-espace de $V$ et s'écrit

$$
\mathbf{E}_{\mathbb{B}_{\nu}}=\frac{1}{\pi} \int_{\mathbb{B}_{\nu}} \omega^{2}\left|\theta_{\nu}(\omega)\right|^{2} \Re e\left\{\mathbf{T}^{S}(\omega)^{*} \mathbf{M}^{S} \mathbf{T}^{S}(\omega)\right\} d \omega
$$

où $\Re$ e désigne la partie réelle, où $\mathbf{T}^{S}(\omega)^{*}$ est l'opérateur adjoint de $\mathbf{T}^{S}(\omega)$ et où $\mathbf{M}^{S}$ est l'opérateur linéaire de masse associé à la forme sesquilinéaire $m^{S}(\mathbf{u}, \mathbf{v})$. On démontre de plus que l'opérateur $\mathbf{E}_{\mathbb{B}_{\nu}}$ admet un ensemble dénombrable de valeurs propres positives décroissantes, chacune ayant une multiplicité $\mathrm{f}$ nie, $\lambda_{1} \geq \lambda_{2} \geq \ldots \rightarrow 0$. Les fonctions propres correspondantes $\left\{\mathbf{e}_{\alpha}\right\}_{\alpha \geq 1}$ telles que

$$
\mathbf{E}_{\mathbb{B}_{\nu}} \mathbf{e}_{\alpha}=\lambda_{\alpha} \mathbf{e}_{\alpha}
$$

sont des fonctions $\mathbf{e}_{\alpha}(\mathbf{x})$ de $\Omega$ dans $\mathbb{R}^{3}$ et constituent une famille complète dans l'espace admissible $V$, orthonormales pour le produit scalaire $\ll \mathbf{u}, \mathbf{v} \gg=\int_{\Omega_{S}} \mathbf{u}(\mathbf{x}, \omega) \cdot \overline{\mathbf{v}(\mathbf{x}, \omega)} d \mathbf{x}$. De plus la série des valeurs propres est sommable $\sum_{\alpha=1}^{+\infty} \lambda_{\alpha}<+\infty$ et sa trace s'écrit

$$
\operatorname{tr} \mathbf{E}_{\mathbb{B}_{\nu}}=\sum_{\alpha=1}^{+\infty} \lambda_{\alpha}<+\infty
$$

Soit $\left\{\mathbf{b}_{1}, \ldots, \mathbf{b}_{n}\right\}$ une base éléments $\mathrm{f}$ nis relative à un maillage éléments $\mathrm{f}$ nis du domaine $\Omega_{S}$ et soit $V_{n}$ le sous-espace engendré par cette base (nous avons noté $n$ et non pas $n_{S}$ le nombre de DDL de la structure af $n$ de simplif er les notations). La projection $\mathbf{E}_{\mathbb{B}_{\nu}, n}$ de l'opérateur $\mathbf{E}_{\mathbb{B}_{\nu}}$ sur $V_{n}$ s'écrit

$$
\mathbf{E}_{\mathbb{B}_{\nu}, n} \mathbf{f}=\sum_{\alpha, \beta=1}^{n}\left[E_{n}\right]_{\alpha \beta} \ll \mathbf{f}, \mathbf{b}_{\beta} \gg \mathbf{b}_{\alpha}
$$

où $\left[E_{n}\right]$ est la matrice réelle $(n \times n)$ symétrique telle que

$$
\begin{gathered}
{\left[E_{n}\right]=\int_{\mathbb{B}_{\nu}}\left[e_{n}(\omega)\right] d \omega,} \\
{\left[e_{n}(\omega)\right]=\frac{1}{\pi} \omega^{2}\left|\theta_{\nu}(\omega)\right|^{2} \Re e\left\{\left[T^{S}(\omega)\right]^{*}\left[M^{S}\right]\left[T^{S}(\omega)\right]\right\} .}
\end{gathered}
$$




\subsubsection{Problème généralisé aux valeurs propres de l'approximation de dimension f nie de l'opérateur d'énergie}

Chaque fonction propre $\mathbf{e}_{\nu}^{n}$ telle que $\mathbf{E}_{\mathbb{B}_{\nu}, n} \mathbf{e}_{\nu}^{n}=\lambda_{\nu}^{n} \mathbf{e}_{\nu}^{n}$ s'écrit $\mathbf{e}_{\nu}^{n}=\sum_{\alpha=1}^{n} P_{\alpha}^{\nu} \mathbf{b}_{\alpha}$. En introduisant le vecteur $\mathbf{P}^{\nu}=\left(P_{1}^{\nu}, \ldots, P_{n}^{\nu}\right)$, on voit que les valeurs propres $\left\{\lambda_{1}^{n}, \ldots, \lambda_{n}^{n}\right\}$ et les vecteurs propres associés $\left\{\mathbf{P}^{1}, \ldots, \mathbf{P}^{n}\right\}$ sont les solutions du problème généralisé aux valeurs propres,

$$
[H] \mathbf{P}=\lambda^{n}[G] \mathbf{P}
$$

avec $[G]$ et $[H]$ des matrices rélles $(n \times n)$ symétriques déf nies positives telles que

$$
[G]_{\beta \alpha}=\ll \mathbf{b}_{\alpha}, \mathbf{b}_{\beta} \gg \quad, \quad[H]=[G]\left[E_{n}\right][G]
$$

Les vecteurs propres $\left\{\mathbf{P}^{1}, \ldots, \mathbf{P}^{n}\right\}$ constituent une base de $\mathbb{R}^{n}$ et vérif ent les propriétés d'orthogonalité suivantes, $\ll \mathbf{e}_{\nu}^{n}, \mathbf{e}_{\nu^{\prime}}^{n} \gg=\mathbf{P}^{\nu^{\prime}}[G] \mathbf{P}^{\nu}=\delta_{\nu \nu^{\prime}}$ et $\mathbf{P}^{\nu^{T}}[H] \mathbf{P}^{\nu}=\delta_{\nu \nu^{\prime}} \lambda_{\nu}^{n}$.

\subsubsection{Construction du modèle matriciel réduit MF adaptée à la band $\mathbb{B}_{\nu}$}

Soit $[P]$ la matrice réelle $(n \times N)$ dont les colonnes sont les $N$ vecteurs propres $\left\{\mathbf{P}^{1}, \ldots, \mathbf{P}^{N}\right\}$ qui correspondent aux $N$ plus grandes valeurs propres $\lambda_{1}^{n} \geq \ldots \geq \lambda_{N}^{n}$. Le modèle matriciel réduit MF de dimension $N$ relatif à la bande $\mathbb{B}_{\nu}$ s'obtient en projetant l'Eq. [124] sur $\left\{\mathbf{P}^{1}, \ldots, \mathbf{P}^{N}\right\}$ et s'écrit

$$
\left[\mathcal{A}_{N}^{S}(\omega)\right] \mathbf{q}^{S}(\omega)=\mathcal{F}_{S}(\omega)
$$

avec

$$
\begin{gathered}
\mathbf{q}^{S}(\omega)=[P] \mathbf{U}(\omega), \\
{\left[\mathcal{A}_{N}^{S}(\omega)\right]=[P]^{T}\left[A^{S}(\omega)\right][P],} \\
\mathcal{F}_{S}(\omega)=[P]^{T} \mathbf{F}_{S}(\omega) .
\end{gathered}
$$

\subsubsection{Méthode de construction du sous-espace propre dominant}

Nous donnons ci-après les éléments principaux de la méthode qui permet d'éviter la construction explicite de la matrice $\left[E_{n}\right]$ (en effet, rappelons que cette matrice de dimension $(n \times n)$ est pleine dans le cadre de la méthode des éléments f nis, alors que la matrice $\left[A^{S}(\omega)\right]$ est creuse).

On utilise la méthode d'itération dans les sous-espaces pour résoudre le problème spectral déf ni par l'Eq. [135]. Soit $m$ la dimension du sous-espace pour les itérations, qui est telle que $N<m \ll n$ (en pratique [BAT 76], on prend $m=\min \{2 N, N+8\})$. Comme l'algorithme usuel de la méthode d'itération dans les sous-espaces est adapté au calcul des plus petites valeurs propres (et non pas des plus grandes), le problème spectral initial déf ni par l'Eq. [135] est reformulé comme suit : calculer les $N$ plus petites valeurs propres et les vecteurs propres associés du problème généralisé symétrique aux valeurs propres,

$$
[G][S]=[H][S][\Gamma],
$$

où $[S]$ est la matrice réelle $(n \times m)$ et [ $\Gamma]$ est la matrice diagonale réelle $(m \times m)$ telles que $[S]^{T}[H][S]=[I]$ et $[S]^{T}[G][S]=[\Gamma]$. On a alors les relations suivantes $[\Lambda]=[\Gamma]^{-1}$ et $[\widetilde{P}]=[S][\Gamma]^{-1 / 2}$, où $[\Lambda]$ est une matrice diagonale réelle $(m \times m)$ et les $N$ plus grandes valeurs propres cherchées sont telles que $\lambda_{1}^{n}=[\Lambda]_{11} \geq \ldots \geq \lambda_{N}^{n}=[\Lambda]_{N N}$ et où $[\widetilde{P}]$ est la matrice réelle $(n \times m)$ dont les $N$ premières colonnes sont les vecteurs propres $\mathbf{P}^{1}, \ldots, \mathbf{P}^{N}$ qui déf nissent la matrice $[P]$. En examinant l'algorithme d'itération dans les sous-espaces, on voit que la matrice $\left[E_{n}\right]$ n'est utilisée qu'une seule fois à chaque iteration pour calculer une matrice $[W]$ réelle $(n \times m)$ telle que

$$
[W]=\left[E_{n}\right][X],
$$

où $[X]$ est une matrice réelle $(n \times m)$ connue. La méthode présentée ci-après permet de calculer $[W]$ connaissant $[X]$ sans avoir à calculer explicitement la matrice $\left[E_{n}\right]$. 
Procédure indirecte dans le domaine fréquentiel. La matrice $[X]$ étant réelle, la matrice $[W]$ peut s'écrire

$$
[W]=\int_{\mathbb{B}_{\nu}} \Re e\{[\widehat{Z}(\omega)]\} d \omega
$$

où $[\widehat{Z}(\omega)]$ est une matrice complexe $(n \times m)$ qui est la solution du système linéaire

$$
\begin{aligned}
& {\left[A^{S}(\omega)\right][\widehat{Y}(\omega)]=\widehat{\chi}(\omega)[X],} \\
& {\left[A^{S}(\omega)\right][\widehat{Z}(\omega)]=\left[M^{S}\right] \overline{[\widehat{Y}(\omega)]},}
\end{aligned}
$$

avec $\widehat{\chi}(\omega)$ la fonction déf nie sur $\mathbb{R}$ par $\widehat{\chi}(\omega)=\frac{1}{\pi} \omega^{2}\left|\theta_{\nu}(\omega)\right|^{2} \mathbf{1}_{\mathbb{B}_{\nu}}(\omega)$. Cette procédure requiert $N_{\text {freq }} \gg 1$ factorisations de la matrice $\left[A^{S}(\omega)\right]$, où $N_{\text {freq }}$ est le nombre de points de fréquence de la bande $\mathbb{B}_{\nu}$ nécessaire pour estimer la fonction $\omega \mapsto\left[T^{S}(\omega)\right]$ sur la bande $\mathbb{B}_{\nu}$.

Procédure indirecte dans le domaine temporel. La procédure est basée sur l'utilisation de la méthode MF présentée au paragraphe 6.4 pour résoudre les Eqs. [144] et [145] sur la bande $\mathbb{B}_{\nu}$, qui ne requiert alors que la factorisation d'une seule matrice complexe $(n \times n)$ (qui est creuse dans le cas de la méthode des éléments f nis), au lieu de $N_{\text {freq }} \gg 1$ factorisations. Soit $\chi_{0}(t)$ la fonction telle que $\chi_{0}(t)=e^{-i \Omega_{\nu} t} \chi(t)$ avec $\chi(t)=(1 / 2 \pi) \int_{\mathbb{B}_{\nu}} e^{i \omega t} \widehat{\chi}(\omega) d \omega$. On en déduit que la transformée de Fourier $\widehat{\chi}_{0}(\omega)=\int_{\mathbb{R}} e^{-i \omega t} \chi_{0}(t) d t$ est telle que $\widehat{\chi}_{0}(\omega)=0$ si $\omega \notin \mathbb{B}_{0}$ où $\mathbb{B}_{0}$ est la bande basse fréquence associée à la bande $\mathbb{B}_{\nu}$, déf nie par l'Eq. [104]. La fonction $\chi_{0}(t)$ est le signal BF associé au signal MF $\chi(t)$. Soient $\left[D_{\nu}^{S}\right]$ et $\left[K_{\nu}^{S}\right]$ les matrices symétriques $(n \times n)$ telles que

$$
\left[D_{\nu}^{S}\right]=\left[D^{S}\left(\Omega_{\nu}\right)\right],\left[K_{\nu}^{S}\right]=\left[K^{S}\left(\Omega_{\nu}\right)\right]
$$

Alors la matrice $[W]$ telle que $[W]=\left[E_{n}\right][X]$ est donnée par la formule

$$
[W]=2 \pi \Re e\left\{\left[Z_{0}(0)\right]\right\},
$$

où $\left[Z_{0}(t)\right]$ est la solution du problème d'évolution basse fréquence suivant

$$
\begin{aligned}
{\left[M^{S}\right]\left[\ddot{Y}_{0}(t)\right]+\left[\widetilde{D}_{\nu}^{S}\right]\left[\dot{Y}_{0}(t)\right]+\left[\widetilde{K}_{\nu}^{S}\right]\left[Y_{0}(t)\right] } & =\chi_{0}(t)[X], \\
{\left[M^{S}\right]\left[\ddot{Z}_{0}(t)\right]+\left[\widetilde{D}_{\nu}^{S}\right]\left[\dot{Z}_{0}(t)\right]+\left[\widetilde{K}_{\nu}^{S}\right]\left[Z_{0}(t)\right] } & =\left[M^{S}\right] \overline{\left[Y_{0}(-t)\right]},
\end{aligned}
$$

avec $\left[\widetilde{D}_{\nu}^{S}\right]$ et $\left[\widetilde{K}_{\nu}^{S}\right]$ les matrices complexes $(n \times n)$ symétriques déf nies par

$$
\left[\widetilde{D}_{\nu}^{S}\right]=\left[D_{\nu}^{S}\right]+2 i \Omega_{\nu}\left[M^{S}\right] \quad, \quad\left[\widetilde{K}_{\nu}^{S}\right]=-\Omega_{\nu}^{2}\left[M^{S}\right]+i \Omega_{\nu}\left[D_{\nu}^{S}\right]+\left[K_{\nu}^{S}\right] .
$$

Il est à noter que les transformées de Fourier $\left[\widehat{Y}_{0}(\omega)\right]$ et $\left[\widehat{Z}_{0}(\omega)\right]$ de $\left[Y_{0}(t)\right]$ et $\left[Z_{0}(t)\right]$ respectivement, sont telles que $\left[\widehat{Y}_{0}(\omega)\right]=\left[\widehat{Z}_{0}(\omega)\right]=[0]$ quand $\omega \notin \mathbb{B}_{0}$. C'est pour cette raison que les Eqs. [148] et [149] correspondent à un problème d'évolution $\mathrm{BF}$ (associé au problème MF initial dans la bande $\mathbb{B}_{\nu}$ ) et peuvent donc être résolues par un schéma d'intégration temporel implicite inconditionnellement stable du type Newmark (voir les commentaires sur la méthode de résolution à la $\mathrm{f} n$ du paragraphe 6.4). On procède donc comme suit (on reprend les notations de la $\mathrm{f}$ du paragraphe 6.4):

Etape 1. Construction de la suite f nie $\left[Y_{0}(i \times \Delta t)\right]$ pour $i=m_{\mathrm{i}}, \ldots, m_{\mathrm{f}}$ en résolvant l'Eq. [148] avec la condition initiale $\left[Y_{0}\left(t_{\mathrm{i}}\right)\right]=\left[\dot{Y}_{0}\left(t_{\mathrm{i}}\right)\right]=[0]$ (on rappelle que $m_{\mathrm{i}}$ est un entier négatif).

Etape 2. Construction de la suite f nie $\left[Z_{0}(j \times \Delta t)\right]$ pour $j=-m_{\mathrm{f}}, \ldots, 0$ en résolvant l'Eq. [149] avec la condition initiale $\left[Z_{0}\left(-t_{\mathrm{f}}\right)\right]=\left[\dot{Z}_{0}\left(-t_{\mathrm{f}}\right)\right]=[0]$.

\section{Conclusion}

Nous avons présenté des modélisations mécaniques et des méthodes numériques avancées et innovantes qui permettent de résoudre les problèmes de vibrations et de vibroacoustique de systèmes complexes en basses et en moyennes fréquences. Ces méthodes ont été conçues et validées en vue du développement des codes de calcul de vibrations et de vibroacoustique de nouvelles générations. 


\section{Bibliographie}

[AMI 90] Amini S., HARRis P.J., ' A comparison between various boundary integral formulations of the exterior acoustic problem ', Comp. Meth. Appl. Mech. Eng., vol. 84, 1990, p. 59-75.

[AMi 92] Amini S., Harris P.J., Wilton D.T., Coupled Boundary and Finite Element Methods for the Solution of the Dynamic Fluid-Structure Interaction Problem, Lecture Notes in Eng., vol. 77, Springer, New York, 1992.

[ANG 83] Angelini J.J., Hutin P.M., ' Exterior Neumann problem for Helmholtz equation. Problem of irregular frequencies `, La Recherche Aérospatiale (English edition), vol. 3, 1983, p. 43-52.

[ANG 92] Angelini J.J., Soize C., Soudais P., 'Hybrid numerical method for solving the harmonic Maxwell equations: II. Construction of the numerical approximations ", La Recherche Aérospatiale (English edition), vol. 4, 1992, p. 45-55.

[BAT 76] Bathe K.J., Wilson E.L., Numerical Methods in Finite Element Analysis, Prentice Hall, New York, 1976.

[BRE 92] Brebila C.A., Dominguez J., Boundary Elements: An Introductory Course, McGraw-Hill, New York, 1992.

[BUR 71] Burton A.J., Miller G.F.,' The application of integral equation methods to the numerical solution of some exterior boundary value problems `, Proc. R. Soc. London Ser. A., vol. 323, 1971, p. 201-210.

[CHE 92] Chen G., Zhou J., Boundary Element Methods, Academic Press, New York, 1992.

[COL 92] Colton D.L., Kress R., Integral Equation Methods in Scattering Theory, Krieger Publishing Company, Malabar, Florida, 1992.

[DAU 92] Dautray R., Lions J.L., Mathematical Analysis and Numerical Methods for Science and Technology, Springer-Verlag, Berlin, 1992.

[HAC 95] HackBusch W., Integral Equations, Theory and Numerical Treatment, Birkhauser Verlag, Basel, 1995.

[MOR 95] Morand H., Ohayon R., Fluid Structure Interaction, Wiley, New York, 1995.

[NED 76] NEDELEC J.C., ' Curved f nite element methods for the solution of singular integral equations of surfaces in 3D , Comp. Meth. Appl. Mech. Eng., vol. 8, 1976, p. 61-80.

[OHA 87] OHAYON R., ' Fluid-structure modal analysis. New symmetric continuum-based formulations. Finite element applications `, Proc. Int. Conf. NUMETA 87, Martinus Sijhoff Publ., The Netherlands, 1987.

[OHA 90] Ohayon R., Felippa C.A.,' The effect of wall motion on the governing equations of contained fuids ", Journal of Applied Mechanics, vol. 57, 1990, p. 783-785.

[OHA 98] Ohayon R., Soize C., Structural Acoustics and Vibration, Academic Press, London, San Diego, 1998.

[PAN 65] PANich O.I., ' On the question of solvability of the exterior boundary value problems for the wave equation and Maxwell's equations `, Russian Math. Surv., vol. 20, 1965, p. 221-226.

[SCH 68] Schenck H.A.,' Improved integral formulation for acoustic radiation problems `J. Acoust. Soc. Am., vol. 44, 1968, p. 41-58.

[SOI 82] SOIZE C., ' Medium frequency linear vibrations of anisotropic elastic structures `, La Recherche Aérospatiale (English edition), vol. 8, 1982, p. 65-87.

[SOI 92] Soize C., Desanti A., David J.M., ' Numerical methods in elastoacoustics for low and medium frequency ranges `, La Recherche Aérospatiale (English edition), vol. 5, 1992, p. 25-44.

[SOI 98] Solze C., ' Reduced models in the medium frequency range for general external structural-acoustics systems ", J. Acoust. Soc. Am., vol. 103, no 6, 1998, p. 3393-3406. 